\title{
Morfometría y dimorfismo sexual de Elasmoderus wagenknechti (Liebermann) (Orthoptera: Tristiridae) en dos eventos de irrupción poblacional
}

\author{
Morphometry and sexual dimorphism of Elasmoderus wagenknechti \\ (Liebermann) (Orthoptera: Tristiridae) in two population outbreaks
}

\author{
JORGE CEPEDA-PIZARRO ${ }^{1}$, SOLANGE VEGA ${ }^{1}$, HERNÁN VÁSQUEZ ${ }^{1} \&$ MARIO ELGUETA $^{2}$ \\ ${ }^{1}$ Departamento de Biología, Universidad de La Serena, La Serena, Chile; \\ e-mail: jcepeda@userena.cl \\ ${ }^{2}$ Sección Entomología, Museo Nacional de Historia Natural Casilla 787, \\ Santiago, Chile; e-mail: melgueta@mnhn.cl
}

\begin{abstract}
RESUMEN
La ecología poblacional de los insectos de secano ha sido poco estudiada en Chile. Existen diferentes especies que habitan los interfluvios de la región desértico transicional que, bajo ciertas condiciones ambientales, irrumpen demográficamente. Una de estas especies es Elasmoderus wagenknechti (Orthoptera: Tristiridae). Esta es una especie endémica a Chile, su rango de distribución se extiende desde Taltal en la Segunda Región ( $25^{\circ} 22^{\prime}$ S , $70^{\circ} 31^{\prime}$ O) hasta Salamanca en la Cuarta Región ( $31^{\circ} 46^{\prime}$ S, $\left.70^{\circ} 58^{\prime} \mathrm{O}\right)$. En este trabajo se comunican y discuten los resultados de análisis morfométricos realizados en individuos adultos de E. wagenknechti. Estos fueron capturados durante dos eventos de irrupción poblacional ocurridos en 1996 y 1999 en la Cuarta Región (Coquimbo, Chile). Elasmoderus wagenknechti ha sido descrita como una especie braquíptera. En este estudio, la relación longitud del tegmen/longitud del abdomen fue 0,66 para el macho y 0,51 para la hembra. Para el ala metatorácica, esta relación fue 0,34 y 0,24 respectivamente. La variabilidad morfométrica de los 23 caracteres examinados resultó baja tanto en el macho (CV: 3,04-31,46 \%) como en la hembra (3,68-32,00\%). A pesar del aspecto más grueso de la hembra, la relación ancho corporal/longitud total fue similar en ambos sexos $(0,27)$. Del mismo modo, tanto en el macho como en la hembra, el nivel de hidratación bordeó el $71 \%$ de la biomasa medida en fresco. En esta misma dirección, la relación biomasa seca/biomasa fresca fue similar en ambos sexos (e.g., 0,28 en el macho; 0,29 en la hembra). Las comparaciones morfométricas entre machos de diferente evento demográfico mostraron 10 diferencias significativas; las hembras, ocho diferencias. Además del tamaño (e.g., longitud total), el dimorfismo sexual quedó evidenciado en doce comparaciones morfométricas. La diferencia favoreció a la hembra en todos los casos. Los índices variable morfométrica o $\%$ / variable morfométrica $0^{\top} 0^{\top}$ más altos correspondieron al $\mathrm{P}_{\mathrm{s}}(2,83), \mathrm{L}_{\mathrm{t}}(1,56), \mathrm{An}_{\text {mes }}(1,55)$ y $\mathrm{L}_{\mathrm{pn}}(1,52)$, todos ellos relacionados al tamaño. El índice de condición fue ligeramente más alto en la hembra $\left(\mathrm{b}_{1}: 1,42\right)$ que en el macho $\left(b_{1}: 1,25\right)$. Aunque esta diferencia no resultó estadísticamente significativa, un valor mayor del índice de condición de las hembras sugeriría una mejor adecuación biológica de estas respecto de los machos. Esta habilidad de la hembras explicaría la capacidad de E. wagenknechti de irrumpir demográficamente en el semidesierto de Chile, una vez dadas las condiciones ambientales favorables para ello.
\end{abstract}

Palabras clave: morfometría de insectos, insectos de secano, Orthoptera, irrupciones poblacionales.

\begin{abstract}
The population ecology of rangeland insects has been little studied in Chile. There are different species inhabiting the inter-valley areas of the transitional desert that, under certain environmental conditions, display population outbreaks. One of them is Elasmoderus wagenknechti (Orthoptera: Tristiridae). It is an endemic species to Chile. Its geographic range of distribution is from Taltal in the Second Region $\left(25^{\circ} 22^{\prime} \mathrm{S}, 70^{\circ} 31^{\prime} \mathrm{W}\right)$ to Salamanca in the Fourth Region ( $\left.31^{\circ} 46^{\prime} \mathrm{S}, 70^{\circ} 58^{\prime} \mathrm{W}\right)$. The results of morphometric analyses carried out on individuals captured from two population outbreaks are reported and discussed in this work. The outbreaks took place in 1996 and 1999 in the Fourth Región (Coquimbo, Chile). Elasmoderus wagenknechti has been described as a brachypterous species. In this work, the relation length of the tegmen/length of the abdomen was 0.66 for the male and 0.51 for the female. To the metathoracic wing, this relation was 0.34 and 0.24 respectively. The morphometric variability of 23 characters examined in this work was low both in the male (CV: 3.04-31.46\%) and in the female (CV: 3.68-32.00\%). Despite the thicker aspect of the female, the relation body width/total length was similar in both sexes (0.27). Likewise, the level of hydration was close to
\end{abstract}


$71 \%$ of fresh biomass in both the male and the female. In the same way, the relation dry biomass/fresh biomass was also similar in both sexes (male: 0.28; female: 0.29). The morphometric comparisons from different outbreaks showed 10 significant differences in males and eight significant differences in females. Besides body size (e.g., total length), the sexual dimorphism showed up in 12 morphometric comparisons. In all of them, the difference was favorable to the female. The highest indices of morphometric variable $9+1$ $0^{\top} \sigma^{\top}$ were dry weight (2.83), total length (1.56), mesonotal width (1.55), and pronotal length (1.52); all of them related to body size. The condition index was higher in the female $\left(b_{1}: 1.42\right)$ as compared to that of the male $\left(b_{1}: 1.25\right)$. Although this difference was not statistically significant, a higher value would suggest a better fitness of the female. This fact might explain the ability of this species to demographically outburst in the Chilean semidesert, once the right environmental conditions have been triggered.

Key words: insect morphometry, rangeland insects, Orthoptera, population outbreaks.

\section{INTRODUCCIÓN}

Las langostas de la familia Tristiridae (Orthoptera: Acridoidea) constituyen un grupo de insectos que habitan principalmente el dominio andino-patagónico (sensu Cabrera \& Willink 1973) de Argentina, Chile y Perú (Cigliano 1989, 1991, Cigliano et al. 1989). Los tristíridos son langostas de tamaño mediano a pequeño, de coloración opaca y en general parduzca o grisácea, con su tegumento rugoso, tuberculado o carenado. Son en su gran mayoría ápteros, exceptuando los representantes de los géneros Elasmoderus y Enodisomacris. Los caracteres que definen a la familia se basan fundamentalmente en algunos aspectos de morfología externa y estructuras genitales del macho y de la hembra (Cigliano 1989). A la fecha, la familia Tristiridae está constituida por 18 géneros; de estos, 11 están representados en Chile (Elgueta et al. 1999).

Elasmoderus Saussure es un género endémico a Chile y está constituido por tres especies: E. lutescens (Blanchard), E. wagenknechti (Liebermann) y E. minutus Cigliano, Ronderos \& Kemp. Sus representantes se distribuyen mayoritariamente en el Norte Chico, entre los $28^{\circ}$ y los $32^{\circ} \mathrm{S}$ (Cigliano 1989, Cigliano et al. 1989, Elgueta et al. 1999). Estas especies comparten caracteres tales como: ojos subcirculares, presencia de carena transversa en la cabeza a nivel del ocelo medio, borde posterior del pronoto proyectado medialmente hacia atrás y conformando un ángulo agudo, fémur posterior con carena dorsal y ventral muy expandidas, además de un notorio dimorfismo sexual reflejado en su tamaño corporal.

Descrita originalmente como Philippiacris wagenknechti (Liebermann, 1954), E. wagenk$n e c h t i$ se caracteriza por ser una especie de tamaño pequeño a mediano, micróptera, con tégmenes que no entran dorsalmente en contacto al estar las alas en reposo, y por poseer una coloración amarillenta en la cara interna de las tibias y fémures posteriores (Cigliano 1989, Ci- gliano et al. 1989). Esta especie ha sido citada erróneamente por Moroni (1972) como E. rabiosus y por Toro (1972) como P. rabiosus. Elgueta et al. (1999) señalan que el rango de distribución geográfica de E. wagenknechti se extiende desde el sur de la Región de Antofagasta (Taltal, $25^{\circ} 22^{\prime} \mathrm{S}, 70^{\circ} 31^{\prime} \mathrm{O}$ ) hasta el sur de la Región de Coquimbo (Salamanca, 31 ${ }^{\circ} 46^{\prime}$ $\mathrm{S}, 70^{\circ} 58^{\prime} \mathrm{O}$ ).

Las poblaciones de E. wagenknechti, especie conocida localmente como "langosta de Combarbalá", irrumpen demográficamente en los ecosistemas de interfluvio del Norte Chico cada ciertos años (Moroni 1972, Toro 1972). La memoria colectiva de la gente de la zona de Combarbalá $\left(31^{\circ} 10^{\prime} \mathrm{S}, 71^{\circ} 00^{\prime} \mathrm{O}\right)$ recuerda aumentos demográficos de E. wagenknechti ocurridos desde 1940. Antecedentes a ser publicados (Cepeda \& Vásquez 1999) ${ }^{1}$ muestran que estas irrupciones poblacionales se expresan focalizadamente en el área, algunos sitios son más favorables a ellas que otros. Esta variabilidad se extiende también a entre eventos (Cepeda et al. 2000) $)^{2}$. Eventos irruptivos que tuvieron lugar en 1996 y 1999 permitieron estudiar y describir con detalle algunas características morfométricas de los adultos de E. wagenknechti. Al respecto nos preguntamos ¿cuáles son las características morfométricas de los adultos de machos y hembras en condiciones de irrupción poblacional? y ¿cuál es su variabilidad entre sitios de un mismo evento y entre eventos? Nuestra hipótesis de trabajo fue que existe un efecto de sitio y evento en las características morfométricas de los adultos de machos y hem-

\footnotetext{
${ }^{1}$ CEPEDA J \& H VÁSQUEZ (1999) Observaciones sobre una irrupción poblacional de Elasmoderus wagenknechti (Orthoptera: Tristiridae). XXI Congreso Nacional de Entomología, Arica, Chile, Libro de Resúmenes: 14.

${ }^{2}$ CEPEDA J, S VEGA \& J PIZARRO (2000) Irrupciones poblacionales de Elasmoderus wagenknechti (Orthoptera: Tristiridae). XXII Congreso Nacional de Entomología, Valdivia, Chile, Libro de Resúmenes: 27.
} 
bras de E. wagenknechti en condiciones de irrupción poblacional. Los objetivos del trabajo fueron: (1) describir morfométricamente los machos y las hembras de E. wagenknechti en condiciones de irrupción poblacional, (2) evaluar el dimorfismo sexual y (3) reunir antecedentes que permitan someter a prueba la hipótesis anterior.

\section{MATERIALES Y MÉTODOS}

Área de estudio

Los especímenes de E. wagenknechti (Fig. 1) sobre los cuales se basó el estudio fueron capturados en la zona de Combarbalá (Provincia de Limarí, Cuarta Región Coquimbo, Chile, Fig. 2), en los poblados (sitios en adelante) de Agua
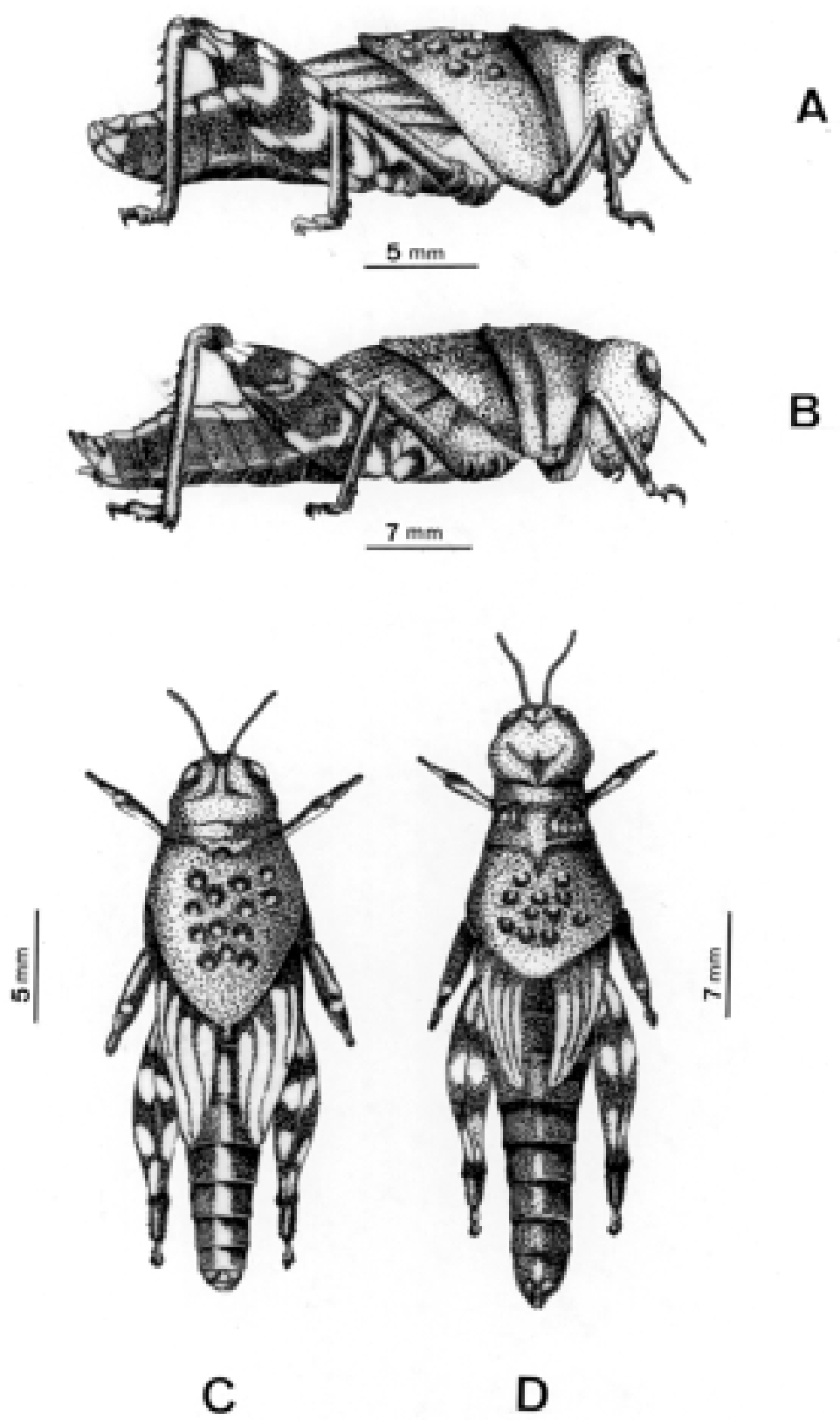

Fig. 1: Aspecto general del adulto de Elasmoderus wagenknechti (Liebermann) (Orthoptera: Tristiridae). (A-C): macho $\left(\mathrm{L}_{\mathrm{t}}, \overline{\mathrm{x}} \pm \mathrm{EE}=25,85 \pm 0,12 \mathrm{~mm}\right)$; $(\mathrm{B}-\mathrm{D})$ : hembra $\left(\mathrm{L}_{\mathrm{t}}, \overline{\mathrm{x}} \pm \mathrm{EE}=40,05 \pm 0,26 \mathrm{~mm}\right)$. 


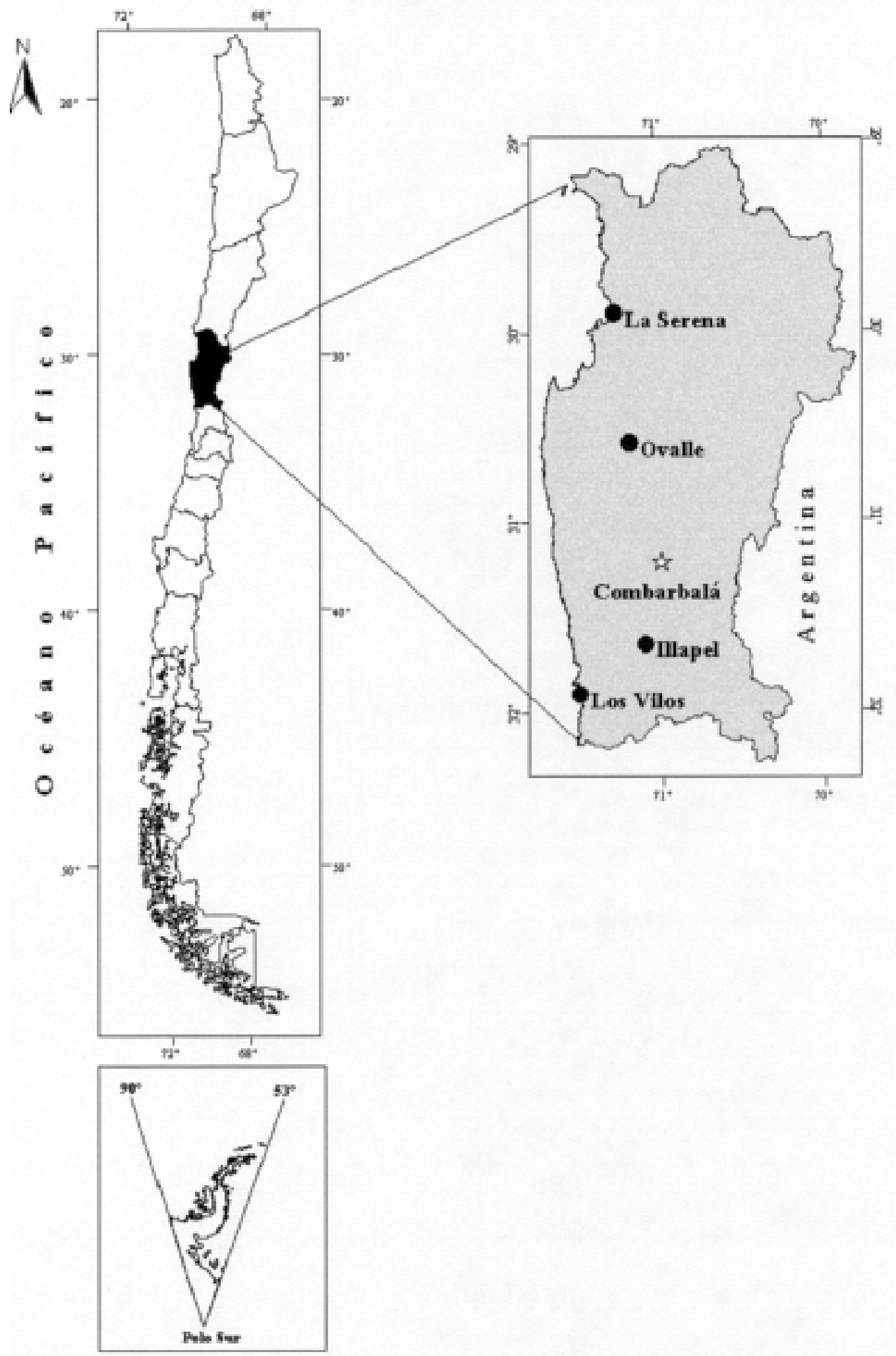

Fig. 2: Mapa del área de estudio (Combarbalá, $31^{\circ} 10^{\prime} \mathrm{S}, 71^{\circ} 00^{\prime} \mathrm{O}$, Cuarta Región Coquimbo, Chile). Map of the study area (Combarbalá, $31^{\circ} 10^{\prime}$ S, $71^{\circ} 00^{\prime}$ W, Fourth Region Coquimbo, Chile). 
Amarilla, El Huacho, La Ciénaga, Quebrada Grande y Lomas del Sauce. Estos sitios fueron escogidos por formar parte del escenario irruptivo en uno (1996) u otro año (1999).

En general, el paisaje del área es discontinuo, producto de la presencia de pendientes suaves en sectores planos o casi planos, entremezcladas con lomajes suaves interrumpidos por pequeñas quebradas y valles menores. Los suelos son de textura gruesa y poco desarrollados, aridisoles (Orthids: Camborthids) (Luzio \& Alcayaga 1992). El clima es de tendencia mediterránea subtropical semiárida (Novoa \& Villaseca 1989). El promedio anual de precipitación es cercano a $210,0 \mathrm{~mm}$, con valores extremos registrados de 21,8-608,2 $\mathrm{mm}$. El valor promedio anual de la temperatura del aire es cercano a $16,5^{\circ} \mathrm{C}(\mathrm{CV}: 19,0 \%)$, con un rango de $12-21{ }^{\circ} \mathrm{C}$. Los meses más fríos son junio y julio $\left(9-12^{\circ} \mathrm{C}\right)$, los más cálidos son enero y febrero $\left(20,0-22,0^{\circ} \mathrm{C}\right)$.

La vegetación nativa es del tipo matorral estepario interior (Gajardo 1993), no recibe influencia directa del mar y ocupa tanto los llanos como las serranías. Entre las especies características se encuentran Adesmia microphylla (adesmia), Atriplex semibaccata (pasto salado), Lithraea caustica (litre), Colliguaja odorifera (colliguaja), Gutierrezia resinosa (pichanilla) y Flourensia thurifera (incienso). Es común la presencia de sectores de laderas ocupados por cultivos de secano, tales como trigo o cebada, eventualmente abandonados, denominados localmente "aguas lluvias" por su dependencia respecto de las precipitaciones. En los huertos caseros se encuentran cultivos de alfalfa, poroto, cebolla, habas, arvejas y frutales. La desertificación en el área es severa por lo que la vegetación original se encuentra muy alterada, persistiendo solo parches degradados de ella (Gastó \& Contreras 1979, Squeo et al. 2002).

\section{Métodos de captura y procesamiento del material}

Los ejemplares se capturaron a mano o con red entomológica en cada uno de los cinco sitios arriba mencionados, en los meses de septiembre y octubre de los años 1996 y 1999. Las capturas se realizaron antes de la aplicación de control químico por el Servicio Agrícola Ganadero. Las recolecciones fueron hechas entre las 10,00 y $16,00 \mathrm{~h}$, durante dos días de cada mes mencionado. Una vez capturados, los insectos fueron depositados en frascos letales de $\mathrm{KCN}$ hasta su procesamiento. Previamente a él, los especímenes fueron depositados en bandejas planas y anchas para su ventilación y posterior selección. Se analizaron solo ejemplares completos y bien conservados.

\section{Estimaciones morfométricas y análisis estadístico}

Se midieron 21 variables morfológicas (en $\mathrm{mm}$ ) y dos de biomasa (peso fresco y peso seco, en $\mathrm{mg}$ ) en machos y hembras adultos. La Tabla 1 muestra los caracteres usados, el criterio de medición y el código que los identifica. En las mediciones se siguió, en general, a Beingolea $(1956,1965,1995)$, Clemente et al. (1987), Cigliano (1989), Cigliano et al. (1989) y Hilliard (2001). Los pesajes se realizaron en una balanza de precisión Accu-

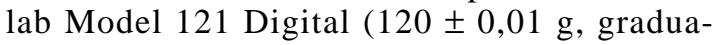
ción $0,01 \mathrm{~g}$ ) en ejemplares frescos y secos. Los ejemplares frescos provinieron directamente de la bandeja de ventilación. El peso seco se logró deshidratando los cuerpos a 50$60{ }^{\circ} \mathrm{C}$ en una estufa Memmert (rango 20-250 ${ }^{\circ} \mathrm{C}$ ), hasta peso constante. Para facilitar el secado, a los ejemplares se les hizo un corte longitudinal en el abdomen siguiendo la línea pleural. Las mediciones lineales se realizaron con un pie de metro Digitimatic (Mitutoyo$500,1 \pm 0,02 \mathrm{~mm})$. Para las dimensiones corporales más finas se utilizó el programa de dominio público IMAGE V 1.67 para Macintosh, desarrollado por el National Institute of Health (htt://rsb.info.nih.gov/nih-image).

Cada una de las variables morfométricas medidas fue descrita en términos de su rango $(\mathrm{R})$, media muestral (x), error estándar (EE) y coeficiente de variación (CV\%). El análisis morfométrico se basó en 202 machos y 198 hembras. El efecto del año del evento (brote en adelante) se midió con la prueba t de Student (para dos muestras independientes, prueba de dos colas). El efecto del sitio (1996: Lomas del Sauce, La Ciénaga y Quebrada Grande; 1999: Lomas del Sauce, La Ciénaga, Agua Amarilla y El Huacho) sobre el tamaño de los individuos (e.g., largo total del cuerpo $\left(\mathrm{L}_{\mathrm{t}}\right)$, peso seco $\left(\mathrm{P}_{\mathrm{s}}\right)$ y peso fresco $\left(\mathrm{P}_{\mathrm{f}}\right)$ se evaluó con análisis de varianza de una entrada (ANDEVA, más de dos muestras, LSD en caso de significación). La relación biomasa seca/largo total del cuerpo se evaluó con regresión lineal $\left(\mathrm{n}_{0^{\top}} 0^{\top}=263, \mathrm{n}\right.$ 우 $=$ 599). La significación estadística de la diferencia entre valores estimados del parámetro $b_{1}$ se evaluó mediante la prueba del intervalo de confianza (Draper \& Smith 1966). En el análisis del dimorfismo sexual se consideraron 202 machos y 198 hembras. Se usaron el parámetro $b_{1}$ como indicador de forma corporal y 13 variables morfométricas: dos de ellas asociadas al tamaño de individuo $\left(\mathrm{P}_{\mathrm{s}} \mathrm{y} \mathrm{L}_{\mathrm{t}}\right)$, cinco a la cabeza 
TABLA 1

Variables morfométricas, códigos y criterios usados en la descripción morfométrica de adultos de Elasmoderus wagenknechti (Liebermann) (Orthoptera: Tristiridae) de dos irrupciones poblacionales en el secano semiárido de Chile

Morphometric variables, codes and criteria used in the morphometric description of adults of Elasmoderus wagenknechti (Liebermann) (Orthoptera: Tristiridae) in two population outbreaks in the semi arid rangeland of Chile

\begin{tabular}{|c|c|c|}
\hline Variable morfométrica & Código & Criterio de medición (en mm si es lineal; en mg si es biomasa) \\
\hline Largo total del cuerpo & $\mathrm{L}_{\mathrm{t}}$ & $\begin{array}{l}\text { Machos: distancia entre el extremo anterior de la cabeza y el ápice de la } \\
\text { placa genital; hembras: distancia entre el extremo anterior de la cabeza y el } \\
\text { extremo del ovipositor }\end{array}$ \\
\hline Ancho máximo de la cabeza & $\mathrm{An}_{\mathrm{mc}}$ & Medida a nivel de las genas (ancho máximo) \\
\hline Altura máxima de la cabeza & $\mathrm{Al}_{\mathrm{mc}}$ & Distancia entre el vértex y el ápice del labro \\
\hline Diámetro vertical del ojo & $\mathrm{Dv}_{\mathrm{o}}$ & Eje vertical máximo del ojo \\
\hline Diámetro horizontal del ojo & $\mathrm{Dh}_{\mathrm{O}}$ & Eje transversal máximo (perpendicular) al eje vertical \\
\hline Ancho del vértice & $\mathrm{An}_{\mathrm{V}}$ & Distancia más corta entre los ojos, en el vértice \\
\hline Longitud del pronoto & $\mathrm{L}_{\mathrm{pn}}$ & Medido a la altura de la carina media pronotal \\
\hline Altura del pronoto & $\mathrm{Al}_{\mathrm{pn}}$ & $\begin{array}{l}\text { Medido como la distancia vertical entre el punto más bajo del lóbulo lateral } \\
\text { del pronoto y el punto más alto de la carina media del mismo, entre el segundo } \\
\text { y tercer surco }\end{array}$ \\
\hline Longitud del esterno & $\mathrm{L}_{\text {est }}$ & $\begin{array}{l}\text { Distancia entre la sutura anterior del esterno y el borde posterior del mismo, } \\
\text { excluyéndose la zona del tubérculo pro esternal }\end{array}$ \\
\hline Ancho del mesoesterno & $\mathrm{An}_{\mathrm{mes}}$ & Medido a nivel de las patas medias \\
\hline Longitud del tegmen & $\mathrm{L}_{\text {teg }}$ & $\begin{array}{l}\text { Distancia lineal entre el extremo distal del tegmen y el margen ventral del } \\
\text { mismo en su unión al pronoto }\end{array}$ \\
\hline Ancho del tegmen & $\mathrm{An}_{\text {teg }}$ & Su lado más ancho \\
\hline Área del tegmen & $\mathrm{Ar}_{\text {teg }}$ & Superfice del tegmen \\
\hline Longitud ala membranosa & $\mathrm{L}_{\mathrm{am}}$ & $\begin{array}{l}\text { Distancia lineal entre el extremo proximal (ángulo humeral) y el extremo } \\
\text { distal, entre el ángulo apical y el margen apical }\end{array}$ \\
\hline Ancho ala membranosa & $\mathrm{An}_{\mathrm{am}}$ & La parte más ancha de la misma \\
\hline Área del ala membranosa & $\mathrm{Ar}_{\mathrm{am}}$ & Superficie del ala membranosa \\
\hline Longitud del fémur posterior & $\mathrm{L}_{\mathrm{fp}}$ & $\begin{array}{l}\text { Medida a lo largo de la cara externa del fémur, desde la unión con el trocánter } \\
\text { hasta el extremo distal del fémur }\end{array}$ \\
\hline Grosor de fémur posterior & $\mathrm{G}_{\mathrm{fp}}$ & Medido en el grosor mayor del fémur \\
\hline Ancho del fémur posterior & $\mathrm{An}_{\mathrm{fp}}$ & Medido en el ancho mayor \\
\hline Longitud del abdomen & $\mathrm{L}_{\mathrm{abd}}$ & Desde su unión al metanoto al extremo distal \\
\hline Ancho del abdomen & $\mathrm{An}_{\mathrm{abd}}$ & Medido en su extremo proximal \\
\hline Peso fresco, peso seco & $\mathrm{P}_{\mathrm{f}}, \mathrm{P}_{\mathrm{s}}$ & En mg, según se describe en el texto \\
\hline
\end{tabular}

$\left(\mathrm{An}_{\mathrm{mc}}, \mathrm{Al}_{\mathrm{mc}}, \mathrm{Dv}_{\mathrm{o}}, \mathrm{Dh}_{\mathrm{o}}, \mathrm{An}_{\mathrm{v}}\right)$, cuatro al tórax $\left(\mathrm{L}_{\mathrm{pn}}, \mathrm{Al}_{\mathrm{pn}}, \mathrm{L}_{\mathrm{est}}, \mathrm{An}_{\mathrm{mes}}\right)$, y dos a las patas posteriores $\left(\mathrm{L}_{\mathrm{fp}}, \mathrm{A}_{\mathrm{nfp}}\right)$. En las comparaciones estadísticas se usaron la prueba $t$ de Student y el intervalo de confianza de $b_{1}$ como se señala más arriba. Los cálculos y las estimaciones de los parámetros se realizaron con el programa Statistix (Anónimo 1996).

\section{RESULTADOS}

\section{Morfometría del macho}

Los caracteres más variables (mayor CV) correspondieron al grosor del fémur posterior $(31,46 \%)$, área del ala membranosa $(29,13 \%)$ y ancho del abdomen $(18,97 \%)$. Los caracteres 
más homogéneos fueron la altura máxima de la cabeza (3,04\%), ancho máximo de la cabeza $(5,20 \%)$, el ancho del mesoesterno $(5,54 \%)$ y la longitud del fémur posterior $(5,57 \%)$. La variabilidad observada y la estimación de los promedios del resto de los caracteres morfológicos examinados se entregan en la Tabla 2.

Expresado como $\mathrm{L}_{\mathrm{t}}$, el tamaño promedio del macho fue 25,64 mm (CV: 5,80\%). En términos de biomasa seca promedio, esta se estimó en 252,85 mg (CV: 14,69 \%). Este valor representa una disminución del $71,52 \%$ respecto de su propio peso fresco promedio $(887,95 \mathrm{mg})$. La cabeza del macho es corta, pero ancha y dilatada en la región mandibular. Su altura promedio $(7,14 \mathrm{~mm})$ es 1,4 veces respecto de su ancho máximo promedio $(5,19 \mathrm{~mm})$. El ojo es pequeño y plano; 1,6 veces más alto $(\overline{\mathrm{x}}: 1,69$ $\mathrm{mm})$ que ancho $(\overline{\mathrm{x}}: 1,09 \mathrm{~mm})$. La distancia inter-ocular o ancho del vértice $(\overline{\mathrm{x}}: 2,22 \mathrm{~mm})$ es 0,4 veces menor que el ancho máximo de la cabeza; dos veces mayor que el ancho del ojo y
1,3 veces su alto. La longitud del pronoto $(\overline{\mathrm{x}}$ : $8,16 \mathrm{~mm})$ es 1,4 veces la altura del mismo $(\overline{\mathrm{x}}$ : $5,63 \mathrm{~mm})$. La relación longitud máxima del esterno $(\overline{\mathrm{x}}: 7,11)$ versus ancho del mesoesterno $(\overline{\mathrm{x}}$ : $7,03 \mathrm{~mm}$ ) es cercana a 1,0. El largo de fémur posterior $(\overline{\mathrm{x}}: 12,21 \mathrm{~mm})$ es 2,4 veces su ancho máximo ( $\overline{\mathrm{x}}: 5,27 \mathrm{~mm})$.

Comparadas entre brotes, 10 de las 13 variables morfológicas estudiadas mostraron diferencias significativas. Las siguientes variables entregaron valores mayores en machos del brote-99 respecto de machos del brote-96: longitud corporal, ancho máximo de la cabeza, ancho del vértice, longitud del pronoto, altura del pronoto, ancho del mesoesterno, longitud del fémur posterior y ancho del fémur posterior. Por el contrario, la biomasa seca promedio y la longitud del esterno fueron mayores en machos del brote-96. En la Tabla 3 se muestra el detalle de las comparaciones realizadas. Aun cuando no se detectaron diferencias significativas entre sitios respecto de $\mathrm{L}_{\mathrm{t}} \mathrm{y}$ biomasa, estas se detectaron en-

TABLA 2

Estimación de parámetros morfométricos de machos adultos de E. wagenknechti: VM: variable morfométrica; $\mathrm{n}=202$; biomasa en $\mathrm{mg}$; otros valores en $\mathrm{mm}^{1}$

Estimates of morphometric parameters of adult males of E. wagenknechti:

$\mathrm{VM}=$ morphometric variable; $\mathrm{n}=202$; biomass in $\mathrm{mg}$; other values in $\mathrm{mm}^{1}$

\begin{tabular}{|c|c|c|c|}
\hline VM & Rango & Media \pm EE & $\mathrm{CV}(\%)$ \\
\hline $\mathrm{L}_{\mathrm{t}}$ & $20,09-30,96$ & $25,64 \pm 0,10$ & 5,80 \\
\hline $\mathrm{P}_{\mathrm{f}}$ & $769,77-921,30$ & $887,95 \pm 7,34$ & 13,41 \\
\hline $\mathrm{P}_{\mathrm{s}}$ & $247,69-266,09$ & $252,85 \pm 2,29$ & 14,69 \\
\hline $\mathrm{An}_{\mathrm{mc}}$ & $4,00-7,20$ & $5,19 \pm 0,02$ & 5,20 \\
\hline $\mathrm{Al}_{\mathrm{mc}}$ & $6,04-8,00$ & $7,14 \pm 0,03$ & 3,04 \\
\hline $\mathrm{Dv}_{\mathrm{o}}$ & $1,40-2,00$ & $1,69 \pm 0,01$ & 6,50 \\
\hline $\mathrm{Dh}_{\mathrm{o}}$ & $0,80-1,40$ & $1,09 \pm 0,01$ & 8,26 \\
\hline $\mathrm{An}_{\mathrm{v}}$ & $1,60-3,00$ & $2,22 \pm 0,01$ & 7,66 \\
\hline $\mathrm{Lp}_{\mathrm{n}}$ & $6,50-9,45$ & $8,16 \pm 0,04$ & 6,49 \\
\hline Alp $_{n}$ & $3,70-6,82$ & $5,63 \pm 0,04$ & 9,41 \\
\hline $\mathrm{L}_{\text {est }}$ & $6,02-8,10$ & $7,11 \pm 0,03$ & 5,62 \\
\hline $\mathrm{An}_{\text {mes }}$ & $6,00-7,93$ & $7,03 \pm 0,03$ & 5,54 \\
\hline $\mathrm{L}_{\text {teg }}$ & $6,93-10,48$ & $8,65 \pm 0,15$ & 8,60 \\
\hline $\mathrm{An}_{\text {teg }}$ & $3,36-4,45$ & $3,78 \pm 0,06$ & 8,20 \\
\hline $\mathrm{Ar}_{\mathrm{teg}}$ & $18,83-33,47$ & $25,73 \pm 0,72$ & 14,30 \\
\hline $\mathrm{L}_{\mathrm{am}}$ & $2,71-5,87$ & $4,46 \pm 0,15$ & 17,26 \\
\hline $\mathrm{An}_{\mathrm{am}}$ & $1,39-2,55$ & $1,85 \pm 0,06$ & 15,13 \\
\hline $\mathrm{Ar}_{\mathrm{am}}$ & $3,11-11,78$ & $6,73 \pm 0,39$ & 29,13 \\
\hline $\mathrm{L}_{\mathrm{fp}}$ & $10,28-13,83$ & $12,21 \pm 0,05$ & 5,57 \\
\hline $\mathrm{G}_{\mathrm{fp}}$ & $0,65-3,01$ & $2,13 \pm 0,13$ & 31,46 \\
\hline $\mathrm{An}_{\mathrm{fp}}$ & $4,10-5,86$ & $5,12 \pm 0,02$ & 6,05 \\
\hline $\mathrm{L}_{\mathrm{abd}}$ & $10,25-15,30$ & $13,01 \pm 0,25$ & 9,53 \\
\hline $\mathrm{An}_{\mathrm{abd}}$ & $3,76-7,29$ & $5,27 \pm 0,20$ & 18,97 \\
\hline
\end{tabular}

${ }^{1}$ Códigos para variables como en la Tabla 1; $\mathrm{Ar}_{\text {teg }}$ y $\mathrm{Ar}_{\mathrm{am}}$ en $\mathrm{mm}^{2}$

Codes for variables as in Table 1; $\mathrm{Ar}_{\text {teg }}$ and $\mathrm{Ar}_{\mathrm{am}}$ in $\mathrm{mm}^{2}$ 
TABLA 3

Morfometría de machos adultos de E. wagenknechti en dos irrupciones poblacionales (1996/1999): VM = variable morfométrica; Biomasa en mg; otros valores en mm; (*) diferencia significativa al $5 \% ;(* *)$ diferencia significativa al $1 \%$ (prueba bilateral t de Student)

Morphometry of adult males of E. wagenknechti in two population outbreaks (1996/1999): VM = morphometric variable; biomass in $\mathrm{mg}$; other numbers in $\mathrm{mm}$;

(*) significant difference at $5 \% ;(* *)$ significant difference at $1 \%$ (two-tailed Student t-test)

\begin{tabular}{|c|c|c|c|c|c|}
\hline \multirow[b]{2}{*}{$\mathrm{VM}^{1}$} & \multirow[b]{2}{*}{ Estadígrafo } & \multicolumn{2}{|c|}{ Año de irrupción } & \multirow[b]{2}{*}{$\underset{t}{\text { Valor de }}$} & \multirow[b]{2}{*}{$\begin{array}{c}\text { Valor de } \\
\mathrm{P}\end{array}$} \\
\hline & & $\begin{array}{c}1996 \\
(n=76)\end{array}$ & $\begin{array}{c}1999 \\
(\mathrm{n}=126)\end{array}$ & & \\
\hline $\mathrm{L}_{\mathrm{t}}$ & $\begin{array}{r}\overline{\mathrm{X}} \pm \mathrm{EE} \\
\text { Rango } \\
\mathrm{CV} \%\end{array}$ & $\begin{array}{r}25,28 \pm 0,18 \\
22,71-30,28 \\
6,32\end{array}$ & $\begin{array}{r}25,85 \pm 0,12 \\
24,22-30,72 \\
7,81\end{array}$ & $-2,60 * *$ & 0,010 \\
\hline $\mathrm{P}_{\mathrm{s}}$ & $\begin{array}{r}\bar{x} \pm \mathrm{EE} \\
\text { Rango } \\
\text { CV\% }\end{array}$ & $\begin{array}{r}280,00 \pm 10,00 \\
140-560 \\
35,71\end{array}$ & $\begin{array}{r}200,40 \pm 15,30 \\
310-400 \\
30,00\end{array}$ & $6,19 * *$ & $<0,0001$ \\
\hline $\mathrm{An}_{\mathrm{mc}}$ & $\begin{array}{r}\overline{\mathrm{X}} \pm \mathrm{EE} \\
\text { Rango } \\
\mathrm{CV} \%\end{array}$ & $\begin{array}{r}5,09 \pm 0,04 \\
4,00-7,20 \\
6,8\end{array}$ & $\begin{array}{r}5,25 \pm 0,02 \\
4,60-5,70 \\
3,62\end{array}$ & $-3,55 * *$ & 0,001 \\
\hline $\mathrm{Al}_{\mathrm{mc}}$ & $\begin{array}{r}\overline{\mathrm{x}} \pm \mathrm{EE} \\
\text { Rango } \\
\mathrm{CV} \%\end{array}$ & $\begin{array}{r}7,11 \pm 0,05 \\
6,10-8,00 \\
6,19\end{array}$ & $\begin{array}{r}7,16 \pm 0,03 \\
6,04-7,97 \\
4,33\end{array}$ & $-0,79$ & 0,430 \\
\hline $\mathrm{Dv}_{\mathrm{o}}$ & $\begin{array}{r}\bar{x} \pm \mathrm{EE} \\
\text { Rango } \\
\mathrm{CV} \%\end{array}$ & $\begin{array}{r}1,70 " 0,01 \\
1,40-1,90 \\
7,14\end{array}$ & $\begin{array}{r}1,69 " 0,01 \\
1,50-2,00 \\
5,32\end{array}$ & $-0,43$ & 0,682 \\
\hline $\mathrm{Dh}_{\mathrm{o}}$ & $\begin{array}{r}\overline{\mathrm{X}} \pm \mathrm{EE} \\
\text { Rango } \\
\mathrm{CV} \%\end{array}$ & $\begin{array}{r}1,10 " 0,01 \\
0,80-1,40 \\
10,90\end{array}$ & $\begin{array}{r}1,08 " 0,01 \\
0,90-1,30 \\
5,56\end{array}$ & 1,79 & 0,076 \\
\hline $\mathrm{An}_{\mathrm{v}}$ & $\begin{array}{r}\overline{\mathrm{X}} \pm \mathrm{EE} \\
\text { Rango } \\
\mathrm{CV} \%\end{array}$ & $\begin{array}{r}2,14 \pm 0,02 \\
0,90-2,50 \\
7,94\end{array}$ & $\begin{array}{r}2,26 \pm 0,01 \\
1,90-3,00 \\
6,64\end{array}$ & $-3,53 * *$ & 0,001 \\
\hline $\mathrm{L}_{\mathrm{pn}}$ & $\begin{array}{r}\overline{\mathrm{X}} \pm \mathrm{EE} \\
\text { Rango } \\
\mathrm{CV} \%\end{array}$ & $\begin{array}{r}8,00 \pm 0,06 \\
6,50-9,30 \\
6,63\end{array}$ & $\begin{array}{r}8,25 \pm 0,05 \\
6,93-9,45 \\
11,0\end{array}$ & $-3,33 * *$ & 0,001 \\
\hline $\mathrm{Al}_{\mathrm{pn}}$ & $\begin{array}{l}\bar{x} \pm \mathrm{EE} \\
\text { Rango }\end{array}$ & $\begin{array}{r}5,48 \pm 0,09 \\
3,70-6,80\end{array}$ & $\begin{array}{r}5,72 \pm 0,03 \\
4,73-6,82\end{array}$ & $-2,57 * *$ & 0,012 \\
\hline CV\% & 13,68 & 5,25 & & & \\
\hline $\mathrm{L}_{\text {est }}$ & $\begin{array}{r}\overline{\mathrm{x}} \pm \mathrm{EE} \\
\text { Rango } \\
\mathrm{CV} \%\end{array}$ & $\begin{array}{r}7,21 \pm 0,05 \\
6,20-8,10 \\
5,68\end{array}$ & $\begin{array}{r}7,04 \pm 0,03 \\
6,02-7,96 \\
5,26\end{array}$ & $2,96 * *$ & 0,004 \\
\hline $\mathrm{An}_{\text {mes }}$ & $\begin{array}{r}\bar{x} \pm \mathrm{EE} \\
\text { Rango } \\
\mathrm{CV} \%\end{array}$ & $\begin{array}{r}6,88 \pm 0,04 \\
6,00-7,50 \\
5,67\end{array}$ & $\begin{array}{r}7,12 \pm 0,03 \\
6,15-7,93 \\
5,20\end{array}$ & $-4,42 * *$ & $<0,0001$ \\
\hline $\mathrm{L}_{\mathrm{fp}}$ & $\begin{array}{r}\overline{\mathrm{x}} \pm \mathrm{EE} \\
\text { Rango } \\
\mathrm{CV} \%\end{array}$ & $\begin{array}{r}11,93 \pm 0,09 \\
10,28-13,71 \\
6,37\end{array}$ & $\begin{array}{r}12,37 \pm 0,05 \\
10,66-13,83 \\
4,52\end{array}$ & $-4,37 * *$ & $<0,0001$ \\
\hline $\mathrm{An}_{\mathrm{fp}}$ & $\begin{array}{r}\bar{x} \pm \text { EE } \\
\text { Rango } \\
\text { CV\% }\end{array}$ & $\begin{array}{r}5,03 \pm 0,04 \\
4,10-5,70 \\
6,96\end{array}$ & $\begin{array}{r}5,17 \pm 0,02 \\
4,10-5,86 \\
5,22\end{array}$ & $-3,07 * *$ & 0,003 \\
\hline
\end{tabular}

${ }^{1}$ Códigos para variables como en la Tabla 1

Codes for variables as in Table 1 
tre años del brote. Así, los machos del brote-99 resultaron más grandes $(26,71 \pm 0,29 \mathrm{~mm})$ que los machos del brote- $96(25,03 \pm 0,25 \mathrm{~mm})$. Esta diferencia se repitió con la biomasa fresca, pero no con la biomasa seca (Tabla 4). Aunque el $\mathrm{R}^{2}$ del ajuste de regresión $\mathrm{P}_{\mathrm{s}} / \mathrm{L}_{\mathrm{t}}$ para machos de $E$. wagenknechti resultó bajo en algunos casos, el modelo alométrico dio cuenta satisfactoria de la relación $\mathrm{P}_{\mathrm{s}}: \mathrm{L}_{\mathrm{t}}$ en ocho de las 10 situaciones examinadas (Tabla 5, P < 0,05). El modelo resultó insatisfactorio con el material procedente de Quebrada Grande y de Lomas del Sauce, ambos del brote-96. En los casos de ajuste satisfactorio, el coeficiente de condición $\left(b_{1}\right)$ tomó valores entre 1,25-2,34. Esta variabilidad se muestra gráficamente en la Fig. 3.

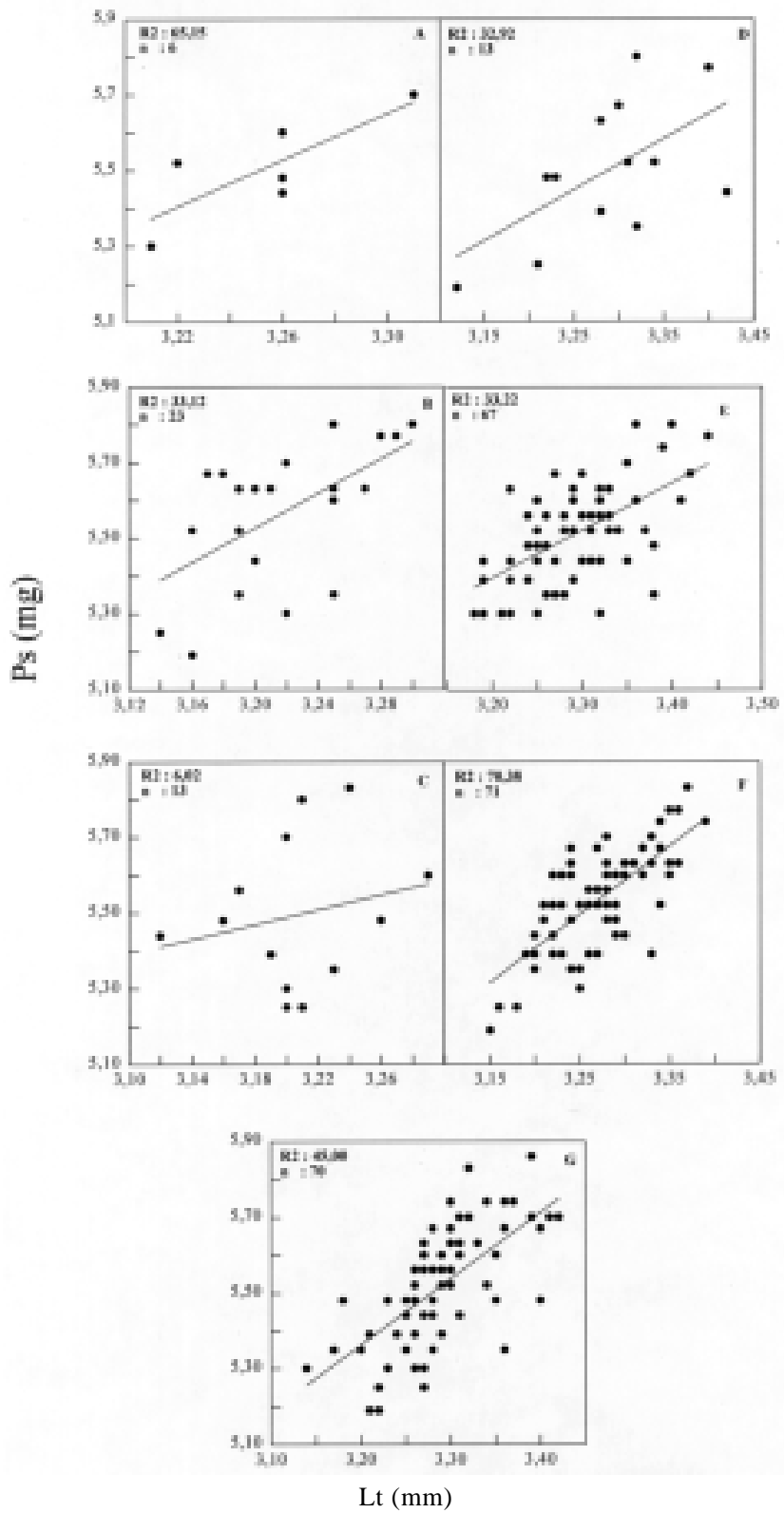

Fig. 3: Relaciones parciales entre peso seco $\left(\mathrm{P}_{\mathrm{s}}, \mathrm{mg}\right)$ y longitud corporal $\left(\mathrm{L}_{\mathrm{t}}, \mathrm{mm}\right)$ del macho adulto de E. wagenknechti. Serie 1996: (A): Lomas del Sauce; (B): La Ciénaga; (C): Quebrada Grande. Serie 1999: (D): Lomas del Sauce; (E): La Ciénaga; (F): Agua Amarilla; (G): El Huacho (para detalles ver Tabla 5).

Partial relationships between dry weight $\left(\mathrm{P}_{\mathrm{s}}, \mathrm{mg}\right)$ and body length $\left(\mathrm{L}_{\mathrm{t}}, \mathrm{mm}\right)$ of adult male of E. wagenknechti. Series 1996: (A): Lomas del Sauce; (B): La Ciénaga; (C): Quebrada Grande. Serie 1999: (D): Lomas del Sauce; (E): La Ciénaga; (F): Agua Amarilla; (G): El Huacho (for further details see Table 5). 


\section{TABLA 4}

Comparación entre sitios de $\mathrm{L}_{\mathrm{t}}, \mathrm{P}_{\mathrm{s}}$ y $\mathrm{P}_{\mathrm{f}}$ de machos adultos de E. wagenknechti en dos eventos de irrupción poblacional en la región semiárida de Chile; $\mathrm{L}_{\mathrm{t}}$ en $\mathrm{mm}$; biomasa en mg; CV en \%

Comparison among sites of $\mathrm{L}_{\mathrm{t}}, \mathrm{P}_{\mathrm{s}}$ and $\mathrm{P}_{\mathrm{f}}$ de adult males of E. wagenknechti in two events of population outbreaks in the semiarid region of Chile. $\mathrm{L}_{\mathrm{t}}$ in $\mathrm{mm}$; biomass in $\mathrm{mg}$; $\mathrm{CV}$ in $\%$

\begin{tabular}{lrccccrr}
\hline Evento & $\mathrm{n}$ & $\mathrm{L}_{\mathrm{t}} \pm \mathrm{EE}$ & $\mathrm{CV}$ & $\mathrm{P}_{\mathrm{f}} \pm \mathrm{EE}$ & $\mathrm{CV}$ & $\mathrm{P}_{\mathrm{s}} \pm \mathrm{EE}$ & $\mathrm{CV}$ \\
\hline 1996 & & & & & & \\
Lomas del Sauce & 6 & $25,89 \pm 0,37 \mathrm{a}$ & 3,51 & $801,67 \pm 6,54 \mathrm{a}$ & 12,00 & $248,33 \pm 14,0 \mathrm{a}$ & 13,81 \\
La Ciénaga & 23 & $25,01 \pm 0,23 \mathrm{a}$ & 4,36 & $804,35 \pm 24,8 \mathrm{a}$ & 14,82 & $266,09 \pm 9,23 \mathrm{a}$ & 16,72 \\
Quebrada Grande & 13 & $24,70 \pm 0,29 \mathrm{a}$ & 4,25 & $760,77 \pm 28,2 \mathrm{a}$ & 13,36 & $247,69 \pm 13,9 \mathrm{a}$ & 20,26 \\
& & & & & & & \\
1999 & 13 & $26,85 \pm 0,60 \mathrm{a}$ & 8,01 & $870,00 \pm 40,05 \mathrm{a}$ & 16,60 & $248,46 \pm 12,7 \mathrm{a}$ & 18,51 \\
Lomas del Sauce & 67 & $26,94 \pm 0,20 \mathrm{a}$ & 5,94 & $921,79 \pm 11,93 \mathrm{a}$ & 10,60 & $249,55 \pm 3,84 \mathrm{a}$ & 12,60 \\
La Ciénaga & 71 & $26,31 \pm 0,17 \mathrm{a}$ & 5,32 & $884,08 \pm 13,89 \mathrm{a}$ & 13,24 & $254,37 \pm 4,04 \mathrm{a}$ & 13,37 \\
Agua Amarilla & 70 & $26,86 \pm 0,18 \mathrm{a}$ & 5,62 & $921,29 \pm 13,38 \mathrm{a}$ & 12,15 & $252,29 \pm 4,66 \mathrm{a}$ & 15,47 \\
El Huacho & & & & & & & \\
& & & & & & & \\
Entre eventos & & & & & & \\
1996 & 42 & $25,03 \pm 0,25$ & $4,04 \mathrm{a}$ & $790,48 \pm 19,94$ & $13,40 \mathrm{a}$ & $257,86 \pm 12,37 \mathrm{a}$ & 16,93 \\
1999 & 221 & $26,71 \pm 0,29$ & $6,22 \mathrm{~b}$ & $906,47 \pm 19,81$ & $13,15 \mathrm{~b}$ & $251,90 \pm 6,31 \mathrm{a}$ & 14,98 \\
\hline
\end{tabular}

${ }^{1}$ Valores seguidos por una misma letra no son estadísticamente significativos (prueba LSD; P > 0,05). Los valores del ANDEVA son los siguientes:

Values followed by the same letter are not statistically significant (LSD test, P > 0,05). The AOV-values are as follows:

$\mathrm{L}_{\mathrm{t}}-96: \mathrm{F}_{2,39}=2,62(\mathrm{P}=0,085) ; \mathrm{L}_{\mathrm{t}}-99: \mathrm{F}_{3,217}=2,28(\mathrm{P}=0,079) ; \mathrm{L}_{\mathrm{t}}, 96-99: \mathrm{F}_{1,261}=43,78(\mathrm{P}<0,0001)$

$\mathrm{P}_{\mathrm{f}}-96 ; \mathrm{F}_{2,39}=0,74(\mathrm{P}=0,483) ; \mathrm{P}_{\mathrm{f}}-99: \mathrm{F}_{3,217}=2,25(\mathrm{P}=0,083) ; \mathrm{P}_{\mathrm{f}}, 96-99: \mathrm{F}_{1,261}=38,22(\mathrm{P}<0,0001)$

$\mathrm{P}_{\mathrm{s}}-96 ; \mathrm{F}_{2,39}=0,85(\mathrm{P}=0,436) ; \mathrm{P}_{\mathrm{s}}-99: \mathrm{F}_{3,217}=0,25(\mathrm{P}=0,859) ; \mathrm{P}_{\mathrm{s}}, 96-99: \mathrm{F}_{1,261}=0,91(\mathrm{P}=0,342)$

\section{TABLA 5}

Valores paramétricos $\left(\mathrm{b}_{0}, \mathrm{~b}_{1}\right)$ y $\mathrm{R}^{2}(\%)$ de la regresión $\left(\mathrm{L}_{\mathrm{n}}\right) \mathrm{P}_{\mathrm{s}} / \mathrm{L}_{\mathrm{t}}$ de machos adultos de E. wagenknechti en dos eventos de irrupción poblacional: (*) regresión significativa al $5 \%$;

$(* *)$ regresión significativa al $1 \%$

Parametric values $\left(\mathrm{b}_{\mathrm{o}}, \mathrm{b}_{1}\right)$ and $\mathrm{R}^{2}(\%)$ of the $\left(\mathrm{L}_{\mathrm{n}}\right)$ linear regression $\mathrm{W}_{\mathrm{d}} / \mathrm{L}_{\mathrm{t}}$ of adult males of $E$. wagenknechti in two population outbreaks: $(*)$ significant regression at $5 \%$; (**) significant regression at $1 \%$

\begin{tabular}{lccccccc}
\hline Análisis & $\mathrm{n}$ & $\mathrm{b}_{\mathrm{o}}$ & $\mathrm{b}_{1}$ & $\mathrm{EE} \mathrm{de} \mathrm{b}_{1}$ & $\mathrm{R}^{2}$ & Valor de F & Valor de P \\
\hline Total & 263 & 4,18 & 1,25 & 0,13 & 26,28 & 93,02 & $<0,0001^{* *}$ \\
& & & & & & & \\
Evento & 42 & 0,76 & 1,81 & 0,58 & 19,75 & 9,85 & $0,003^{*}$ \\
1996 & 221 & 1,90 & 1,49 & 0,13 & 37,13 & 129,34 & $<0,0001^{* *}$ \\
1999 & & & & & & &
\end{tabular}

Sitios x Evento

1996

Lomas del Sauce

La Ciénaga

Quebrada Grande

1999

Lomas del Sauce

La Ciénaga

Agua Amarilla

El Huacho

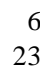

23

13

13

67

71

70

$\begin{array}{ll}0,01 & 3,19 \\ 0,14 & 2,34 \\ 6,42 & 1,13\end{array}$

1,17

0,73

1,35

3,35

1,30

1,22

0,56

0,22

0,66

1,82

0,78

1,75

0,22

0,26
7,48

10,40

0,70

5,40

32,33

70,38

45,00
0,052

$0,004 * *$

0,410 


\section{Morfometría de la hembra}

La variabilidad de los caracteres de la hembra se muestra en la Tabla 6. Los caracteres más variables (mayor CV) fueron el grosor del fémur posterior $(32,00 \%)$, área del ala membranosa $(27,43 \%)$, longitud del ala membranosa $(27,47$ $\%)$ y peso seco $(19,12 \%)$. A su vez, los caracteres menos variables resultaron el ancho y el alto máximo de la cabeza $(3,68$ y $3,95 \%$ respectivamente). Se detectaron diferencias significativas entre años del brote en ocho de las 14 variables morfológicas estudiadas (Tabla 7, prueba t de Student, $\mathrm{P}<0,05)$. Las siguientes variables fueron más grandes en hembras del brote-96 respecto de hembras del brote-99: longitud corporal, peso seco, longitud del pronoto, longitud del esterno, ancho del mesoesterno y ancho del fémur posterior. En el sentido opuesto, se obtuvieron valores menores con el ancho máximo de la cabeza y el diámetro horizontal del ojo.

Expresado como $\mathrm{L}_{\mathrm{t}}$, el tamaño promedio de la hembra fue 40,05 mm (CV: 9,14\%). En términos de biomasa seca promedio, la hembra pesó 778,00 mg (CV: 19,12\%). Este valor representa una disminución del $71,04 \%$ respecto de la biomasa fresca promedio $(2686,59 \mathrm{mg})$. La cabeza de la hembra es subrectangular, siendo la altura $(\overline{\mathrm{x}}: 9,88 \mathrm{~mm}) 1,4$ veces mayor que su respectivo ancho máximo $(\overline{\mathrm{x}}: 7,06 \mathrm{~mm})$. El ojo compuesto es 1,6 veces más alto $(\overline{\mathrm{x}}: 1,93$ $\mathrm{mm})$ que su ancho $(\overline{\mathrm{x}}: 1,20 \mathrm{~mm})$. La distancia interocular o ancho del vértice $(\overline{\mathrm{x}}: 3,08 \mathrm{~mm})$ es 0,4 veces menor que el ancho máximo promedio de la cabeza; 1,6 veces mayor que el alto del ojo y 2,6 veces mayor que el ancho. La longitud del pronoto $(\overline{\mathrm{x}}: 12,44 \mathrm{~mm})$ es $1,6 \mathrm{ve}$ ces la altura del mismo $(\overline{\mathrm{x}}: 8,03 \mathrm{~mm})$. La rela-

TABLA 6

Estimación de parámetros morfométricos de hembras adultas de E. wagenknechti: $\mathrm{VM}=$ variable morfométrica; $\mathrm{n}=198$; biomasa en $\mathrm{mg}$; otros valores en $\mathrm{mm}^{1}$

Estimates of morphometric parameters of adult females of $E$. wagenknechti: $\mathrm{VM}=$ morphometric variable; $\mathrm{n}=198$; biomass in $\mathrm{mg}$; other values in $\mathrm{mm}^{1}$

\begin{tabular}{|c|c|c|c|}
\hline VM & Rango & Media \pm EE & $\mathrm{CV}(\%)$ \\
\hline $\mathrm{L}_{\mathrm{t}}$ & $31,24-49,61$ & $40,05 \pm 0,26$ & 9,14 \\
\hline$P_{f}$ & $2460,91-2932,10$ & $2686,59 \pm 15,73$ & 14,22 \\
\hline Ps & $650,78-872,73$ & $778,00 \pm 6,08$ & 19,12 \\
\hline $\mathrm{An}_{\mathrm{mc}}$ & $6,30-7,80$ & $7,06 \pm 0,02$ & 3,68 \\
\hline $\mathrm{Al}_{\mathrm{mc}}$ & $8,77-10,83$ & $9,88 \pm 0,03$ & 3,95 \\
\hline $\mathrm{Dv}_{\mathrm{o}}$ & $1,50-2,20$ & $1,93 \pm 0,01$ & 6,21 \\
\hline $\mathrm{Dh}_{\mathrm{o}}$ & $0,80-1,50$ & $1,20 \pm 0,01$ & 10,83 \\
\hline $\mathrm{An}_{\mathrm{v}}$ & $2,50-4,00$ & $3,08 \pm 0,02$ & 7,14 \\
\hline $\mathrm{Lp}_{\mathrm{n}}$ & $8,40-14,28$ & $12,44 \pm 0,06$ & 6,67 \\
\hline $\mathrm{Alp}_{\mathrm{n}}$ & $6,64-9,55$ & $8,03 \pm 0,03$ & 5,60 \\
\hline $\mathrm{L}_{\text {est }}$ & $8,52-11,81$ & $10,24 \pm 0,04$ & 6,15 \\
\hline $\mathrm{An}_{\text {mes }}$ & $9,35-18,83$ & $10,89 \pm 0,06$ & 7,44 \\
\hline $\mathrm{L}_{\text {teg }}$ & $9,90-12,95$ & $11,09 \pm 0,13$ & 5,95 \\
\hline $\mathrm{An}_{\text {teg }}$ & $4,59-7,46$ & $5,70 \pm 0,11$ & 5,95 \\
\hline $\mathrm{Ar}_{\text {teg }}$ & $35,69-75,81$ & $49,49 \pm 1,56$ & 15,76 \\
\hline $\mathrm{L}_{\mathrm{am}}$ & $3,66-6,86$ & $5,24 \pm 0,51$ & 27,47 \\
\hline $\mathrm{An}_{\mathrm{am}}$ & $1,78-2,88$ & $2,21 \pm 0,06$ & 13,57 \\
\hline $\mathrm{Ar}_{\mathrm{am}}$ & $5,28-14,69$ & $9,21 \pm 0,51$ & 27,47 \\
\hline $\mathrm{L}_{\mathrm{fp}}$ & $10,06-18,50$ & $16,37 \pm 0,07$ & 6,47 \\
\hline $\mathrm{G}_{\mathrm{fp}}$ & $0,65-3,40$ & $2,50 \pm 0,16$ & 32,00 \\
\hline $\mathrm{An}_{\mathrm{fp}}$ & $5,87-7,81$ & $6,70 \pm 0,03$ & 5,67 \\
\hline $\mathrm{L}_{\mathrm{abd}}$ & $17,53-25,92$ & $21,74 \pm 0,44$ & 10,03 \\
\hline $\mathrm{An}_{\mathrm{abd}}$ & 6,88-11,61 & $9,51 \pm 0,18$ & 9,67 \\
\hline
\end{tabular}

${ }^{1}$ Códigos para variables como en la Tabla $1 ; \mathrm{Ar}_{\text {teg }}$ y $\mathrm{Ar}_{\mathrm{am}}$ en $\mathrm{mm}^{2}$

Codes for variables as in Table 1; $\mathrm{Ar}_{\text {teg }}$ and $\mathrm{Ar}_{\mathrm{am}}$ in $\mathrm{mm}^{2}$ 
TABLA 7

Morfometría de hembras adultas de E. wagenknechti en dos irrupciones poblacionales (1996/1999):

$\mathrm{VM}=$ variable morfométrica; biomasa en $\mathrm{mg}$; otros valores en $\mathrm{mm} ;\left(^{*}\right)$ diferencia significativa al $5 \% ;(* *)$ diferencia significativa al $1 \%$ (prueba bilateral $\mathrm{t}$ de Student) ${ }^{1}$

Morphometry of adult females of E. wagenknechti in two population outbreaks (1996/1999):

$\mathrm{VM}=$ morphometric variable; biomass in $\mathrm{mg}$; other numbers in $\mathrm{mm} ;(*)$ significant difference at $5 \%$;

(**) significant difference at $1 \%$ (two-tailed Student t-test $)^{1}$

\begin{tabular}{|c|c|c|c|c|c|}
\hline \multirow[b]{2}{*}{${ }^{1} \mathrm{VM}$} & \multirow[b]{2}{*}{ Estadígrafo } & \multicolumn{2}{|c|}{ Año de irrupción } & \multirow[b]{2}{*}{ Valor de $\mathrm{t}$} & \multirow[b]{2}{*}{ Valor de $\mathrm{P}$} \\
\hline & & $\begin{array}{c}1996 \\
(\mathrm{n}=64)\end{array}$ & $\begin{array}{c}1999 \\
(\mathrm{n}=134)\end{array}$ & & \\
\hline $\mathrm{L}_{\mathrm{t}}$ & $\begin{array}{c}\overline{\mathrm{X}} \pm \mathrm{EE} \\
\text { Rango } \\
\text { CV\% }\end{array}$ & $\begin{array}{r}42,79 \pm 0,42 \\
34,79-49,61 \\
7,92\end{array}$ & $\begin{array}{r}38,74 \pm 0,26 \\
31,24-49,47 \\
7,77\end{array}$ & $8,16 * *$ & $<0,0001$ \\
\hline $\mathrm{P}_{\mathrm{s}}$ & $\begin{array}{c}\bar{x} \pm \mathrm{EE} \\
\text { Rango } \\
\text { CV\% }\end{array}$ & $\begin{array}{r}0,70 \pm 0,01 \\
0,42-0,92 \\
15,71\end{array}$ & $\begin{array}{r}0,63 \pm 0,01 \\
0,39-0,91 \\
17,46\end{array}$ & $4,25 * *$ & $<0,0001$ \\
\hline $\mathrm{An}_{\mathrm{mc}}$ & $\begin{array}{r}\overline{\mathrm{X}} \pm \mathrm{EE} \\
\text { Rango } \\
\text { CV\% }\end{array}$ & $\begin{array}{r}7,01 \pm 0,04 \\
6,30-7,60 \\
4,14\end{array}$ & $\begin{array}{r}7,09 \pm 0,02 \\
6,40-7,80 \\
3,52\end{array}$ & $-2,04 *$ & $<0,044$ \\
\hline $\mathrm{Al}_{\mathrm{mc}}$ & $\begin{array}{r}\overline{\mathrm{X}} \pm \mathrm{EE} \\
\text { Rango } \\
\mathrm{CV} \%\end{array}$ & $\begin{array}{r}9,92 \pm 0,05 \\
9,12-10,82 \\
4,03\end{array}$ & $\begin{array}{r}9,86 \pm 0,03 \\
8,77-10,83 \\
3,85\end{array}$ & $-0,61$ & 0,543 \\
\hline$D v_{o}$ & $\begin{array}{c}\overline{\mathrm{X}} \pm \mathrm{EE} \\
\text { Rango } \\
\text { CV\% }\end{array}$ & $\begin{array}{r}1,93 \pm 0,02 \\
1,50-2,20 \\
6,74\end{array}$ & $\begin{array}{r}1,94 \pm 0,01 \\
1,60-2,20 \\
5,67\end{array}$ & $-0,51$ & 0,613 \\
\hline $\mathrm{Dh}_{\mathrm{o}}$ & $\begin{array}{r}\overline{\mathrm{X}} \pm \mathrm{EE} \\
\text { Rango } \\
\mathrm{CV} \%\end{array}$ & $\begin{array}{r}1,06 \pm 0,02 \\
0,80-1,50 \\
11,32\end{array}$ & $\begin{array}{r}1,26 \pm 0,01 \\
1,90-1,50 \\
6,35\end{array}$ & $-12,03 * *$ & $<0,0001$ \\
\hline $\mathrm{An}_{\mathrm{v}}$ & $\begin{array}{c}\bar{x} \pm \mathrm{EE} \\
\text { Rango } \\
\text { CV\% }\end{array}$ & $\begin{array}{r}3,07 \pm 0,03 \\
2,50-4,00 \\
7,17\end{array}$ & $\begin{array}{r}3,09 \pm 0,02 \\
2,50-3,70 \\
6,79\end{array}$ & $-0,09$ & 0,931 \\
\hline $\mathrm{Lp}_{\mathrm{n}}$ & $\begin{array}{r}\overline{\mathrm{X}} \pm \mathrm{EE} \\
\text { Rango } \\
\text { CV\% }\end{array}$ & $\begin{array}{r}12,89 \pm 0,08 \\
11,25-14,28 \\
5,07\end{array}$ & $\begin{array}{r}12,23 \pm 0,07 \\
8,40-13,94 \\
6,70\end{array}$ & $6,13 * *$ & $<0,0001$ \\
\hline $\mathrm{Al}_{\mathrm{pn}}$ & $\begin{array}{r}\bar{x} \pm \text { EE } \\
\text { Rango } \\
\text { CV\% }\end{array}$ & $\begin{array}{r}8,08 \pm 0,08 \\
6,91-9,55 \\
7,42\end{array}$ & $\begin{array}{r}8,01 \pm 0,03 \\
6,64-8,97 \\
4,49\end{array}$ & 0,96 & 0,342 \\
\hline $\mathrm{L}_{\text {est }}$ & $\begin{array}{r}\overline{\mathrm{X}} \pm \mathrm{EE} \\
\text { Rango } \\
\mathrm{CV} \%\end{array}$ & $\begin{array}{r}10,55 \pm 0,08 \\
8,52-11,81 \\
6,37\end{array}$ & $\begin{array}{r}10,10 \pm 0,05 \\
8,87-11,50 \\
5,57\end{array}$ & $4,70 * *$ & $<0,0001$ \\
\hline $\mathrm{An}_{\mathrm{mes}}$ & $\begin{array}{r}\overline{\mathrm{x}} \pm \mathrm{EE} \\
\text { Rango } \\
\mathrm{CV} \%\end{array}$ & $\begin{array}{r}11,24 \pm 0,13 \\
9,79-18,83 \\
9,60\end{array}$ & $\begin{array}{r}10,72 \pm 0,05 \\
9,35-12,58 \\
5,32\end{array}$ & $3,61 * *$ & $<0,0001$ \\
\hline $\mathrm{L}_{\mathrm{fp}}$ & $\begin{array}{r}\bar{X} \pm \mathrm{EE} \\
\text { Rango } \\
\text { CV\% }\end{array}$ & $\begin{array}{r}16,29 \pm 0,17 \\
10,06-18,31 \\
8,41\end{array}$ & $\begin{array}{r}16,41 \pm 0,08 \\
13,06-18,50 \\
5,30\end{array}$ & 0,61 & 0,544 \\
\hline $\mathrm{An}_{\mathrm{fp}}$ & $\begin{array}{r}\overline{\mathrm{X}} \pm \mathrm{EE} \\
\text { Rango } \\
\mathrm{CV} \%\end{array}$ & $\begin{array}{r}6,90 \pm 0,04 \\
6,06-7,59 \\
6,96\end{array}$ & $\begin{array}{r}6,60 \pm 0,03 \\
5,87-7,81 \\
5,30\end{array}$ & $5,64 * *$ & $<0,0001$ \\
\hline
\end{tabular}

${ }^{1}$ Códigos para variables como en la Tabla 1

Codes for variables as in Table 1 
ción longitud máxima del esterno ( $\overline{\mathrm{x}}$ : 10,24 $\mathrm{mm})$ versus ancho promedio del mesoesterno $(\overline{\mathrm{x}}: 10,89 \mathrm{~mm})$ es cercana a 1,0. El largo del fémur posterior $(\overline{\mathrm{x}}: 16,37 \mathrm{~mm})$ es 2,4 veces su ancho máximo ( $\overline{\mathrm{x}}: 6,70 \mathrm{~mm})$.

A diferencia del macho, se detectaron algunas diferencias significativas entre sitios respecto del tamaño de la hembra (Tabla 8). En el brote-96, por ejemplo, la hembra promedio del sitio La Ciénaga fue más pequeña $\left(\mathrm{L}_{\mathrm{t}}: 37,65\right.$ $\mathrm{mm})$ que los especímenes capturados en Lomas del Sauce $\left(\mathrm{L}_{\mathrm{t}}: 39,84 \mathrm{~mm}\right)$ y Quebrada Grande $\left(\mathrm{L}_{\mathrm{t}}: 39,95 \mathrm{~mm}\right)$. En el brote-99, la hembra promedio de los sitios Lomas del Sauce $\left(\mathrm{L}_{\mathrm{t}}: 41,32 \mathrm{~mm}\right)$ y La Ciénaga $\left(\mathrm{L}_{\mathrm{t}}: 41,71\right.$ $\mathrm{mm})$ fueron más grandes que aquellas de los sitios Agua Amarilla ( $\mathrm{L}_{\mathrm{t}}: 38,12 \mathrm{~mm}$ ) y El Huacho $\left(\mathrm{L}_{\mathrm{t}}: 38,34 \mathrm{~mm}\right)$. La biomasa no arrojó un patrón consistente. En el brote-96, de las seis comparaciones realizadas, solo la hembra promedio del sitio Quebrada Grande (e.g., 872,73 $\mathrm{mg}$ ) mostró una diferencia significativa respecto del resto. En el brote-99, se detectaron diferencias significativas tanto respecto de la biomasa fresca como seca. La hembra promedio de mayor peso fresco correspondió a especímenes provenientes de La Ciénaga $(2942,10$ $\mathrm{mg}$ ). La hembra promedio de mayor peso seco correspondió a material capturado en Lomas del Sauce (Tabla 8). El valor de $\mathrm{R}^{2}$ del ajuste de regresión alométrica $\mathrm{P}_{\mathrm{s}} / \mathrm{L}_{\mathrm{t}}$ para hembras $E$. wagenknechti fue, en general, más alto que aquel obtenido para machos. El modelo dio cuenta satisfactoria de la relación en todas las situaciones examinadas (Tabla 9, Fig. 4). El coeficiente de condición $\left(b_{1}\right)$ tomó valores entre 1,17-1,79 (Tabla 9).

\section{Dimorfismo sexual}

Elasmoderus wagenkchneti exhibió un marcado dimorfismo sexual. Excepto por $\mathrm{Dh}_{\mathrm{o}}$, variable con la que se obtuvieron resultados contradictorios, la hembra mostró valores absolutos más altos que el macho en el resto de las variable comparadas (Tabla 10). En orden decreciente, los índices $\mathrm{VM}$ 甲 $9 / \mathrm{VM}$ o $^{\star}{ }^{\star}$ más altos correspondieron a Ps, $(2,83)$, Lt $(1,56), \mathrm{An}_{\text {mes }}(1,55)$ y $\mathrm{L}_{\mathrm{pn}}(1,52)$. Todas ellas están relacionadas al tamaño. Los índices menores correspondieron a las dimensiones cefálicas $\mathrm{Dh}_{\mathrm{o}}(1,10)$ y $\mathrm{Dv}_{\mathrm{o}}$ $(1,14)$. Entre brotes, las hembras exhibieron valores morfométricos más altos que los machos, exceptuando Dho (Tabla 11).

TABLA 8

Comparaciones entre sitios de $\mathrm{L}_{\mathrm{t}}, \mathrm{P}_{\mathrm{s}}$ y $\mathrm{P}_{\mathrm{f}}$ de hembras adultas de E. wagenknechti en dos eventos de irrupción poblacional en la región semiárida de Chile: $\mathrm{L}_{\mathrm{t}}$ en mm; $\mathrm{P}_{\mathrm{f}}$ y $\mathrm{P}_{\mathrm{s}}$ en $\mathrm{mg} ; \mathrm{CV}$ en $\%^{1}$

Comparisons among sites of $\mathrm{L}_{\mathrm{t}}, \mathrm{P}_{\mathrm{s}}$ and $\mathrm{P}_{\mathrm{f}}$ de adult females of $E$. wagenknechti in two events of population outbreaks in the semiarid region of Chile: $\mathrm{L}_{\mathrm{t}}$ in $\mathrm{mm} ; \mathrm{P}_{\mathrm{f}}$ and $\mathrm{P}_{\mathrm{s}}$ in $\mathrm{mg} ; \mathrm{CV}$ in $\%{ }^{1}$

\begin{tabular}{lrcrcrrr}
\hline Evento & $\mathrm{n}$ & $\mathrm{L}_{\mathrm{t}} \pm \mathrm{EE}$ & $\mathrm{CV}$ & $\mathrm{P}_{\mathrm{f}} \pm \mathrm{EE}$ & $\mathrm{CV}$ & $\mathrm{P}_{\mathrm{s}} \pm \mathrm{EE}$ & $\mathrm{CV}$ \\
\hline 1996 & & & & & & & \\
Lomas del Sauce & 144 & $39,84 \pm 0,21 \mathrm{a}$ & 6,40 & $2653,40 \pm 27,96 \mathrm{a}$ & 12,64 & $806,46 \pm 8,84 \mathrm{a}$ & 13,16 \\
La Ciénaga & 39 & $37,65 \pm 0,41 \mathrm{~b}$ & 6,85 & $2569,49 \pm 66,80 \mathrm{a}$ & 16,24 & $805,64 \pm 26,96 \mathrm{a}$ & 17,02 \\
Quebrada Grande & 44 & $39,95 \pm 0,46 \mathrm{a}$ & 7,66 & $2629,09 \pm 49,67 \mathrm{a}$ & 12,53 & $872,73 \pm 21,34 \mathrm{~b}$ & 16,22 \\
& & & & & & & \\
1999 & & & & & & & \\
Lomas del Sauce & 136 & $41,32 \pm 0,23 \mathrm{a}$ & 6,41 & $2829,93 \pm 29,82 \mathrm{~b}$ & 12,29 & $846,62 \pm 15,05 \mathrm{a}$ & 20,72 \\
La Ciénaga & 81 & $41,72 \pm 0,40 \mathrm{a}$ & 8,63 & $2932,10 \pm 48,01 \mathrm{a}$ & 14,74 & $734,94 \pm 14,32 \mathrm{~b}$ & 17,53 \\
Agua Amarilla & 78 & $38,12 \pm 0,30 \mathrm{~b}$ & 12,56 & $2556,79 \pm 36,36 \mathrm{c}$ & 12,56 & $708,85 \pm 12,13 \mathrm{~b}$ & 12,56 \\
El Huacho & 77 & $38,34 \pm 0,29 \mathrm{~b}$ & 6,57 & $2460,91 \pm 37,77 \mathrm{c}$ & 13,47 & $650,78 \pm 11,11 \mathrm{c}$ & 14,99 \\
& & & & & & & \\
Entre eventos & & & & & & & 15,47 \\
1996 & 227 & $39,15 \pm 0,74 \mathrm{a}$ & 6,97 & $2617,29 \pm 48,14 \mathrm{a}$ & 13,80 & $829,28 \pm 20,04 \mathrm{a}$ & 15,45 \\
1999 & 372 & $39,87 \pm 0,74 \mathrm{~b}$ & 8,54 & $2694,93 \pm 37,99 \mathrm{~b}$ & 13,26 & $735,30 \pm 13,15 \mathrm{~b}$ & 16,45 \\
\hline
\end{tabular}

${ }^{1}$ Valores seguidos por la misma letra no son estadísticamente significativos (prueba LSD, P > 0,05). Los valores del ANDEVA son los siguientes:

Values followed by the same letter are not statistically significant (LSD test, P > 0.05). The AOV-values are as follows:

$\mathrm{L}_{\mathrm{t}}-96: \mathrm{F}_{2,224}=11,26(\mathrm{P}<0,0001) ; \mathrm{L}_{\mathrm{t}}-99: \mathrm{F}_{3,368}=39,29(\mathrm{P}<0,0001) ; \mathrm{Lt}, 96-99: \mathrm{F}_{1,597}=5,94(\mathrm{P}=0,015)$

$\mathrm{P}_{\mathrm{f}}-96: \mathrm{F}_{2,224}=0,89(\mathrm{P}=0,415) ; \mathrm{P}_{\mathrm{f}}-99: \mathrm{F}_{3,368}=32,33(\mathrm{P}<0,0001) ; \mathrm{P}_{\mathrm{f}}, 96-99: \mathrm{F}_{1,597}=6,81(\mathrm{P}=0,009)$

$\mathrm{P}_{\mathrm{s}}-96: \mathrm{F}_{2,224}=5,51(\mathrm{P}=0,005) ; \mathrm{P}_{\mathrm{s}}-99: \mathrm{F}_{3,368}=37,62(\mathrm{P}<0,0001) ; \mathrm{P}_{\mathrm{s}}, 96-99: \mathrm{F}_{1,597}=29,33(\mathrm{P}<0,0001)$ 
TABLA 9

Valores paramétricos $\left(\mathrm{b}_{\mathrm{o}}, \mathrm{b}_{1}\right)$ y $\mathrm{R}^{2}(\%)$ de la regresión lineal $\left(\mathrm{L}_{\mathrm{n}}\right) \mathrm{P}_{\mathrm{s}} / \mathrm{L}_{\mathrm{t}}$ de hembras adultas de $E$. wagenknechti en dos eventos de irrupción poblacional: $(*)$ regresión significativa al $5 \%$;

(**) regresión significativa al $1 \%$

Parametric estimates $\left(\mathrm{b}_{\mathrm{o}}, \mathrm{b}_{1}\right)$ y $\mathrm{R}^{2}(\%)$ of see linear regression $\left(\mathrm{L}_{\mathrm{n}}\right) \mathrm{W}_{\mathrm{d}} / \mathrm{L}_{\mathrm{t}}$ of adult females of E. wagenknechti in two population outbreaks: $(*)$ significant regression at $5 \%$;

(**) significant regression at $1 \%$

\begin{tabular}{lccccccc}
\hline Análisis & $\mathrm{n}$ & $\mathrm{b}_{\mathrm{o}}$ & $\mathrm{b}_{1}$ & $\mathrm{EE} \mathrm{de} \mathrm{b}_{1}$ & $\mathrm{R}^{2}$ & Valor de F & Valor de P \\
\hline Total & 599 & 4,06 & 1,42 & 0,08 & 33,05 & 294,71 & $<0,0001^{* *}$ \\
Evento & & & & & & & \\
1996 & 227 & 5,99 & 1,34 & 0,11 & 38,63 & 141,63 & $<0,0001^{* *}$ \\
1999 & 372 & 2,29 & 1,57 & 0,10 & 38,34 & 230,09 & $<0,0001^{* *}$
\end{tabular}

Sitios x Evento

1996

Lomas del Sauce $\quad 144 \quad 10,59 \quad 1,17$

Quebrada Grande

1,38

0,14

32,28

67,70

$<0,0001 * *$

$1,19 \quad 1,79$

0,31

46,79

32,53

$<0,0001^{* *}$

1999

Lomas del Sauce 136

La Ciénaga $\quad 81$

$36 \quad 3,0$

Agua Amarilla

$3,06 \quad 1,5$

1,51

1,50

0,22

61,13

66,06

$<0,0001 * *$

El Huacho

$\begin{array}{ll}2,75 & 1,50 \\ 4,66 & 1,38\end{array}$

0,25

21,34

52,75

36,36

$<0,0001^{* *}$

$1,38 \quad 0,21$

36,03

88,18

42,80

$<0,0001 * *$

$\begin{array}{llll}2,64 & 1,51 & 0,20 & 43,22\end{array}$

$<0,0001 * *$

$<0,0001^{* *}$

TABLA 10

Dimorfismo sexual de E. wagenknechti: VM = variable corporal comparada; prueba bilateral t de Student;

(*) diferencia significativa al $5 \% ;(* *)$ diferencia significativa al $1 \% ; \mathrm{n}_{\text {machos }}=202, \mathrm{n}_{\text {hembras }}=198$

Sexual dimorphism in E. wagenknechti: VM = body variable being compared; two tailed Student t-test; (*) significant difference at $5 \% ;(* *)$ significant difference at $1 \% ; \mathrm{n}_{\text {males }}=202, \mathrm{n}_{\text {females }}=198$

\begin{tabular}{|c|c|c|c|c|c|}
\hline $\mathrm{VM}^{1}$ & $\begin{array}{l}\text { Macho } \\
x \pm E E\end{array}$ & $\begin{array}{l}\text { Hembra } \\
x \pm E E\end{array}$ & Valor de $\mathrm{t}$ & Valor de P & Relación $\varphi \varphi / 0^{\pi} 0^{\pi}$ \\
\hline $\mathrm{L}_{\mathrm{t}}$ & $25,64 \pm 0,10$ & $40,05 \pm 0,26$ & $-51,38 * *$ & $<0,0001$ & 1,56 \\
\hline $\mathrm{P}_{\mathrm{s}}$ & $0,23 \pm 0,01$ & $0,65 \pm 0,01$ & $-42,50 * *$ & $<0,0001$ & 2,83 \\
\hline $\mathrm{An}_{\mathrm{mc}}$ & $5,19 \pm 0,02$ & $7,06 \pm 0,02$ & $-70,10 * *$ & $<0,0001$ & 1,36 \\
\hline $\mathrm{Al}_{\mathrm{mc}}$ & $7,14 \pm 0,03$ & $9,88 \pm 0,03$ & $-43,11^{* *}$ & $<0,0001$ & 1,38 \\
\hline$D v_{o}$ & $1,69 \pm 0,01$ & $1,93 \pm 0,01$ & $-21,69 * *$ & $<0,0001$ & 1,14 \\
\hline $\mathrm{Dh}_{\mathrm{o}}$ & $1,09 \pm 0,01$ & $1,20 \pm 0,01$ & $-9,60 * *$ & $<0,0001$ & 1,10 \\
\hline $\mathrm{An}_{\mathrm{v}}$ & $2,22 \pm 0,01$ & $3,08 \pm 0,02$ & $-31,71 * *$ & $<0,0001$ & 1,39 \\
\hline $\mathrm{L}_{\mathrm{pn}}$ & $8,16 \pm 0,04$ & $12,44 \pm 0,06$ & $-61,53 * *$ & $<0,0001$ & 1,52 \\
\hline $\mathrm{Al}_{\mathrm{pn}}$ & $5,63 \pm 0,04$ & $8,03 \pm 0,03$ & $-48,77 * *$ & $<0,0001$ & 1,43 \\
\hline $\mathrm{L}_{\text {est }}$ & $7,11 \pm 0,03$ & $10,24 \pm 0,04$ & $-59,29 * *$ & $<0,0001$ & 1,44 \\
\hline $\mathrm{An}_{\text {mes }}$ & $7,03 \pm 0,03$ & $10,89 \pm 0,06$ & $-60,52^{* *}$ & $<0,0001$ & 1,55 \\
\hline $\mathrm{L}_{\mathrm{fp}}$ & $12,21 \pm 0,05$ & $16,37 \pm 0,07$ & $-46,71 * *$ & $<0,0001$ & 1,34 \\
\hline $\mathrm{An}_{\mathrm{fp}}$ & $5,12 \pm 0,02$ & $6,70 \pm 0,03$ & $-45,83 * *$ & $<0,0001$ & 1,31 \\
\hline
\end{tabular}

${ }^{1}$ Códigos para variables como en la Tabla 1

Codes for variables as in Table 1 

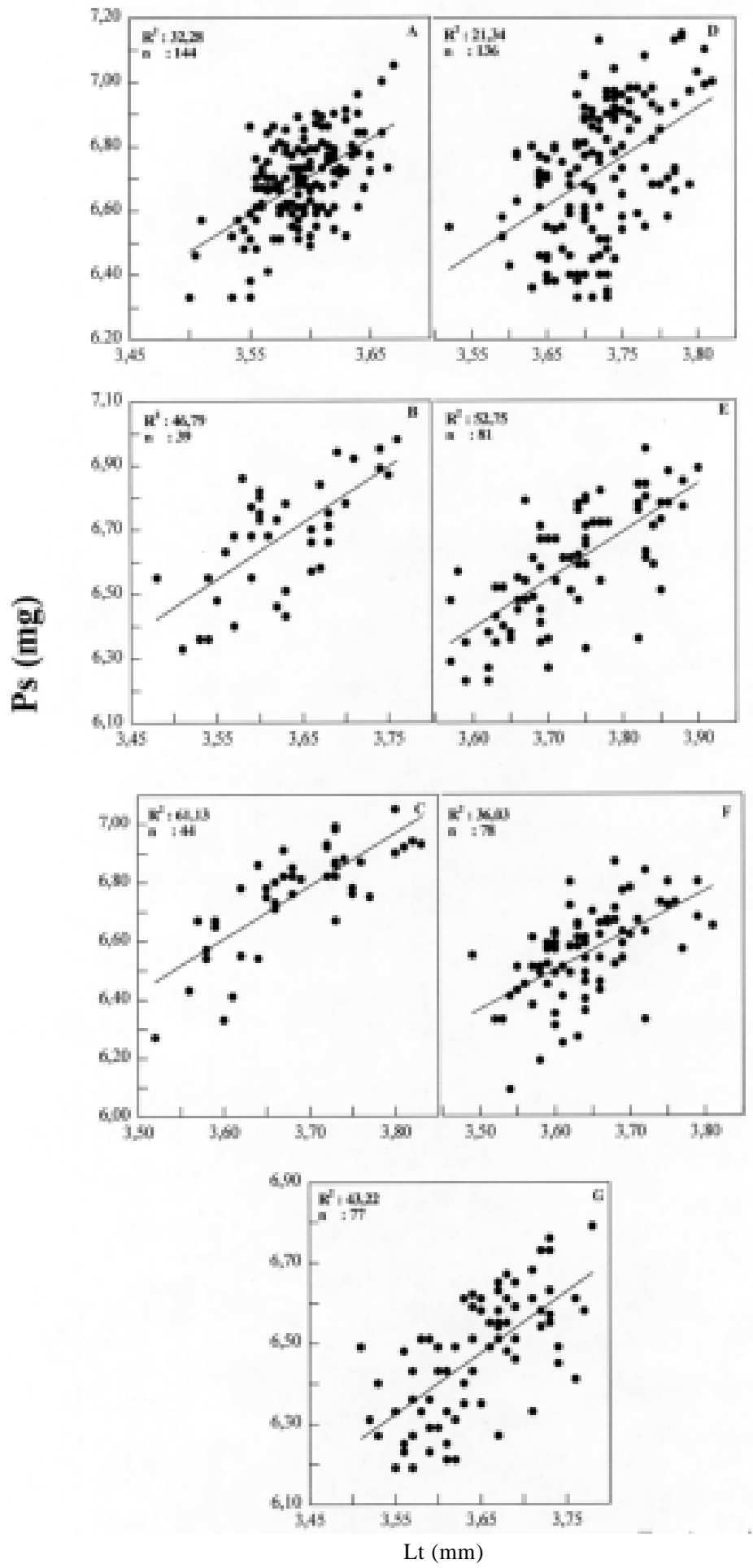

Fig. 4: Relaciones parciales entre peso seco $\left(\mathrm{P}_{\mathrm{s}}, \mathrm{mg}\right)$ y longitud corporal $\left(\mathrm{L}_{\mathrm{t}}, \mathrm{mm}\right)$ de la hembra adulta de E. wagenknechti. Serie 1996: (A): Lomas del Sauce; (B): La Ciénaga; (C): Quebrada Grande. Serie 1999: (D): Lomas del Sauce; (E): La Ciénaga; (F): Agua Amarilla; (G): El Huacho (para detalles ver Tabla 9).

Partial relationships between dry weight $\left(\mathrm{P}_{\mathrm{s}}, \mathrm{mg}\right)$ and body length $\left(\mathrm{L}_{\mathrm{t}}, \mathrm{mm}\right)$ of adult female of E. wagenknechti. Series 1996: (A): Lomas del Sauce; (B): La Ciénaga; (C): Quebrada Grande. Series 1999: (D): Lomas del Sauce; (E): La Ciénaga; (F): Agua Amarilla; (G): El Huacho (for further details see Table 9). 


\section{TABLA 11}

Comparaciones de parámetros morfométricos (media $\pm \mathrm{EE}$ ) de machos y hembras adultas de $E$. wagenknechti según años de irrupción poblacional: $\mathrm{P}_{\mathrm{s}}$ en $\mathrm{mg}$, otros valores en $\mathrm{mm}$; prueba bilateral

$\mathrm{t}$ de Student; brote 1996: $\mathrm{n}_{\text {machos }}=76, \mathrm{n}_{\text {hembras }}=64$; brote 1999: $\mathrm{n}_{\text {machos }}=126, \mathrm{n}_{\text {hembras }}=134$

Comparisons of morphometric parameters (mean $\pm \mathrm{SE}$ ) of adult males and females of E. wagenknechti according to years of population outbreak: $\mathrm{W}_{\mathrm{d}}$ in $\mathrm{mg}$, other values are in $\mathrm{mm}$; two-tailed Student t-test; 1996 outbreak: $\mathrm{n}_{\text {males }}=76, \mathrm{n}_{\text {females }}=$ $64 ; 1999$ outbreak: $\mathrm{n}_{\text {males }}=126, \mathrm{n}_{\text {females }}=134$

\begin{tabular}{|c|c|c|c|c|c|c|c|c|}
\hline $\mathrm{VM}^{1}$ & Macho & $\begin{array}{c}1996 \\
\text { Hembra }\end{array}$ & $\begin{array}{c}\text { Valor de } \\
t\end{array}$ & $\begin{array}{c}\text { Valor de } \\
\mathrm{P}\end{array}$ & Macho & $\begin{array}{c}1999 \\
\text { Hembra }\end{array}$ & $\underset{t}{\text { Valor de }}$ & $\begin{array}{c}\text { Valor de } \\
\text { P }\end{array}$ \\
\hline $\mathrm{L}_{\mathrm{t}}$ & $25,28 \pm 0,18$ & $42,79 \pm 0,42$ & $-37,88$ & $(<0,0001)$ & $25,85 \pm 0,12$ & $38,74 \pm 0,26$ & $-44,88$ & $(<0,0001)$ \\
\hline $\mathrm{P}_{\mathrm{s}}$ & $0,28 \pm 0,01$ & $0,70 \pm 0,01$ & $-23,82$ & $(<0,0001)$ & $0,20 \pm 0,01$ & $0,63 \pm 0,01$ & $-40,74$ & $(<0,0001)$ \\
\hline $\mathrm{An}_{\mathrm{mc}}$ & $5,09 \pm 0,04$ & $7,01 \pm 0,04$ & $-35,29$ & $(<0,0001)$ & $5,25 \pm 0,02$ & $7,09 \pm 0,02$ & $-68,28$ & $(<0,0001)$ \\
\hline $\mathrm{Al}_{\mathrm{mc}}$ & $7,11 \pm 0,05$ & $9,92 \pm 0,05$ & $-15,55$ & $(<0,0001)$ & $7,16 \pm 0,03$ & $9,86 \pm 0,03$ & $-63,26$ & $(<0,0001)$ \\
\hline$D v_{o}$ & $1,70 \pm 0,01$ & $1,93 \pm 0,02$ & $-11,19$ & $(<0,0001)$ & $1,69 \pm 0,01$ & $1,93 \pm 0,01$ & $-19,04$ & $(<0,0001)$ \\
\hline $\mathrm{Dh}_{\mathrm{o}}$ & $1,10 \pm 0,01$ & $1,06 \pm 0,01$ & 2,06 & $(0,0420)$ & $1,08 \pm 0,01$ & $1,26 \pm 0,01$ & $-20,75$ & $(<0,0001)$ \\
\hline $\mathrm{An}_{\mathrm{v}}$ & $2,14 \pm 0,02$ & $3,07 \pm 0,03$ & $-27,14$ & $(<0,0001)$ & $2,26 \pm 0,01$ & $3,09 \pm 0,02$ & $-22,23$ & $(<0,0001)$ \\
\hline $\mathrm{Lp}_{\mathrm{n}}$ & $8,00 \pm 0,06$ & $12,89 \pm 0,08$ & $-48,52$ & $(<0,0001)$ & $8,25 \pm 0,05$ & $12,23 \pm 0,07$ & $-47,12$ & $(<0,0001)$ \\
\hline $\mathrm{Al}_{\mathrm{pn}}$ & $5,48 \pm 0,09$ & $8,08 \pm 0,07$ & $-2,69$ & $(<0,0001)$ & $5,72 \pm 0,03$ & $8,00 \pm 0,03$ & $-55,86$ & $(<0,0001)$ \\
\hline $\mathrm{L}_{\text {est }}$ & $7,21 \pm 0,05$ & $10,55 \pm 0,08$ & $-34,83$ & $(<0,0001)$ & $7,04 \pm 0,03$ & $10,09 \pm 0,05$ & $-51,98$ & $(<0,0001)$ \\
\hline $\mathrm{An}_{\mathrm{mes}}$ & $6,88 \pm 0,04$ & $11,24 \pm 0,13$ & $-30,76$ & $(<0,0001)$ & $7,12 \pm 0,03$ & $10,72 \pm 0,05$ & $-60,70$ & $(<0,0001)$ \\
\hline $\mathrm{L}_{\mathrm{fp}}$ & $11,93 \pm 0,09$ & $16,29 \pm 0,17$ & $-22,66$ & $(<0,0001)$ & $12,37 \pm 0,05$ & $16,41 \pm 0,08$ & $-44,52$ & $(<0,0001)$ \\
\hline $\mathrm{An}_{\mathrm{fp}}$ & $5,03 \pm 0,04$ & $6,90 \pm 0,04$ & $-31,60$ & $(<0,0001)$ & $5,17 \pm 0,02$ & $6,60 \pm 0,03$ & $-36,98$ & $(<0,0001)$ \\
\hline
\end{tabular}

${ }^{1}$ Códigos para variables como en la Tabla 1

Codes for variables as in Table 1

\section{DISCUSIÓN}

El significado de las variaciones morfométricas intrapoblacionales ha sido analizado por diferentes autores (LaBarbera 1989, Reilly \& Wainwright 1994, Ricklefs \& Miles 1994, Wainwright 1994, Koehl 1996). Que los insectos presentan variaciones morfométricas asociadas al ambiente es un fenómeno bien conocido (Daly 1985, Anónimo 1990, Thompson 1992, Palmer 1994, Cepeda-Pizarro et al. 1996, Krasnov et al. 1996, Williams 2001). Las causas de esta variabilidad no son del todo conocidas. Las respuestas fenotípicas dependen tanto de las características ambientales, particularmente de aquellas que actúan durante el desarrollo del individuo, como de las propiedades genéticas de las especies (Daly 1985, Anónimo 1990, Williams 2001).

A diferencia de E. lutescens (Blanchard), para la cual Cigliano (1989) señala una elevada variabilidad intraespecífica respecto de la longitud de las alas y del cuerpo de las hembras, nada se consigna en la literatura en relación a E. wagenknechti y E. minutus. Los resultados encontrados en este estudio muestran que $E$. wagenknechti exhibe una elevada variabilidad morfométrica en condiciones de explosión de- mográfica. Esta variabilidad es tanto espacial como temporal. Estos resultados son concordantes con nuestra hipótesis de trabajo. Las comparaciones morfométricas entre machos de diferente evento irruptivo mostraron 10 diferencias significativas. La mayoría de ellas entregó una diferencia favorable a los individuos del brote-99. Las comparaciones morfométricas entre hembras mostraron ocho diferencias significativas. Seis fueron favorables a los especímenes del evento-96 y dos lo fueron a los ejemplares de evento-99.

Puesto que solo se tiene información de las características morfométricas de los individuos en condiciones de irrupción poblacional, no es posible establecer si esta variabilidad morfométrica es propia del fenómeno irruptivo o es una característica independiente de él. Antecedentes informados en la literatura (Beingolea 1956, 1965, Gage \& Mukerji 1977, Pfadt 1977, 1982, 1984, Joern 1979, Kemp 1992, Cigliano et al. 1995) señalan como probables causas de variabilidad fenotípica a factores asociados tanto a la dinámica de los recursos como a las características meteorológicas del período del evento. Podría ser el caso de E. wagenknechti. En nuestra área de trabajo, aunque los años de los eventos no difirieron en temperatura promedio del aire 
$\left(\sim 17{ }^{\circ} \mathrm{C}\right)$, sí lo hicieron en precipitación anual (1996: $120 \mathrm{~mm}$ de precipitación total anual; 1999: $166 \mathrm{~mm}$, de un rango de precipitación de 21,8-608,2 mm). El acceso a recursos alimentarios de mejor calidad (e.g., los sitios difieren entre sí respecto de la disponibilidad de plantas cultivadas, condición de los suelos y cobertura vegetal) podría ser también causa de estas diferencias. Aspectos que necesitan ser explorados con mayor detención y direccionalidad.

El dimorfismo sexual de E. wagenknechti quedó evidenciado en 13 comparaciones morfométricas. La diferencia fue favorable a la hembra en todas ellas. Los índices $\mathrm{VM}_{\odot \odot} / \mathrm{VM}^{\circ} 0^{\circ}$ más altos correspondieron al $\mathrm{P}_{\mathrm{S}}(2,83), \mathrm{L}_{\mathrm{t}}$ $(1,56)$ y $\mathrm{An}_{\text {mes }}(1,55)$, todos ellos relacionados al tamaño. Existen algunas hipótesis que pueden explicar estas diferencias de tamaño. De acuerdo a la hipótesis de la tolerancia al estrés hídrico (Chappell 1983, Prange \& Pinshow 1994, Rourke 2000), el tamaño más grande de la hembra podría estar relacionado a una mayor tolerancia a la falta de agua que el macho. Esta tolerancia facilitaría tanto el transporte forético que la hembra hace del macho como la búsqueda de alimento y sitios favorables de oviposición. De acuerdo a la hipótesis de fecundidad (Lande 1980, Slatkin 1984, Greenwood \& Adams 1987, Hedrick \& Temeles 1989, Shine 1989, Fairbairn 1990, 1993, 1997, Andersen 1994, Blanckenhorn et al. 1995, Head 1995, Prenter et al. 1995, Fairbairn \& Preziosi 1996, Preziosi \& Fairbairn 1996, 2000, Preziosi et al. 1996, Reeve \& Fairbairn 1999, Villagra et al. 2001, Higgins 2002), el mayor tamaño de la hembra podría estar dirigido a acumular reservas energéticas tendientes a lograr oviposturas elevadas. Opuestamente, para explicar el menor tamaño del macho, algunos autores -entre ellos Parker (1978), Siva-Jothy (1987), Rubenstein 1989, Rowe (1994), Blanckenhorn \& Fairbairn (1995), Jablonski \& Vepsäläine (1995), Arnqvist et al. (1996), Rowe \& Arnqvist (1996), Price (1997), Arnqvist \& Danielsson (1999)han sugerido que esta diferencia estaría destinada a asegurar la fertilización de la hembra. En E. wagenknechti el menor tamaño del macho y su hábito forético podrían estar destinados no solo a asegurar la fertilización de la hembra y así lograr incorporar su material genético al flujo génico de la población, sino también a facilitar en esta la búsqueda del alimento y sitios de oviposición, junto a impedir que otros machos se aparearan con la misma hembra. El dimorfismo sexual también afecta a las proporciones de la cabeza de los individuos de E. wagenknechti. Thompson (1992) mostró una asociación positiva entre el tamaño de la cabeza de Melanoplus femurrubrum y su eficiencia alimentaria. Individuos con cabeza más grande tenían una mayor eficacia. El tamaño de la cabeza se encontró, a su vez, regulado por el tipo de alimento. Según fuera el tipo de alimento, resultaba el tamaño de la cabeza. Para Thompson (1992) esta plasticidad tiene un claro significado adaptativo. Es posible que con E. wagenknechti ocurra una situación parecida.

La relación masa corporal/tamaño o índice de condición evaluada a través del parámetro $b_{1}$ representa una medida de la eficacia biológica de los individuos (Jakob et al. 1996). Esta relación también ha sido usada para evaluar el dimorfismo sexual respecto de la forma corporal (Shine et al. 2002). En este trabajo, $b_{1}$ promedio fue más alto en la hembra $\left(b_{1}: 1,42\right)$ que en el macho $\left(b_{1}: 1,25\right)$. Aunque esta diferencia no resultó significativa $(\mathrm{P}>0,05$; prueba intervalo de confianza al 95\%), este resultado estaría apoyando, de acuerdo a consideraciones ecofisiológicas (Prange \& Pinshow 1994, Lactin 1998, Henschel et al. 2001), la hipótesis de una mayor eficacia biológica de las hembras respecto de los machos.

\section{AGRADECIMIENTOS}

Carlos Zuleta R. y Jaime Pizarro A. del Departamento de Biología de la Universidad de La Serena, Patricia Larraín S. del Instituto de Investigaciones Agropecuarias (INIA-INTIHUASI) y Elizabeth Chiappa de la Universidad de Playa Ancha comentaron una versión preliminar del trabajo. Dos revisores anónimos y el editor de la revista sugirieron valiosas modificaciones editoriales al manuscrito, las que enriquecieron la versión final de este. Estudio financiado por la Dirección de Investigación de la Universidad de La Serena (Proyecto DIULS No. 0220-2-07) a J. Cepeda.

\section{LITERATURA CITADA}

ANDERSEN NM (1994) The evolution of sexual size dimorphism and mating systems in the water striders (Hemiptera: Gerridae): a phylogenetic approach. Ecoscience 1: 208-214.

ANÓNIMO (1990) Locust handbook. Natural Resources Institute, Overseas Development Administration, London, United Kingdom. 202 pp.

ANÓNIMO (1996) Statistix for windows. User's manual. Analytical software. Tallahassee Florida, USA. v + 333 pp.

ARNQVIST G, L ROWE, JJ KRUPA \& A SIH (1996) Assortative mating by size: a meta analysis of mating patterns in water striders systems. Evolutionary Ecology 10: 265-284. 
ARNQVIST G \& I DANIELSSON (1999) Copulatory behavior, genital morphology, and male fertilization success in water striders. Evolution 53: 147-156.

BEINGOLEA O (1956) Estudios morfométricos de fases de la langosta migratoria sudamericana del Perú (Schistocerca cancellata Serv., S. paranensis Burn.). Proceedings of the Tenth International Congress of Entomology 2: 1037-1051.

BEINGOLEA O (1965) Sumario bio-ecológico de la langosta migratoria sudamericana Schistocerca cancellata Serv (S. paranensis Burm.) en el Perú. Revista Peruana de Entomología Agrícola 6: 39-60 (1963).

BEINGOLEA O (1995) Langostas y su control. Editorial RAAA/SENASA, Lima, Perú. 143 pp.

BLANCKENHORN WU \& DJ FAIRBAIRN (1995) Life history adaptation along a latitudinal cline in the water strider Aquarius remigis (Heteroptera: Gerridae). Journal of Evolutionary Biology 8: 21-41.

BLANCKENHORN WU, RF PREZIOSI \& DJ FAIRBAIRN (1995) Time and energy constraints and the evolution of sexual size dimorphism, to-eat or to mate? Evolutionary Ecology 9: 369-381.

CABRERA AL \& A WILLINK (1973) Biogeografía de América Latina. Organización de Estados Americanos, Serie Biología. Monografía 13, Washington, District of Columbia, USA. 120 pp.

CEPEDA-PIZARRO JG, H VÁSQUEZ, H VEAS \& GO COLON (1996) Relaciones entre tamaño corporal y biomasa en adultos de Tenebrionidae (Coleoptera) de la estepa costera del margen meridional del desierto chileno. Revista Chilena de Historia Natural 69: 67-76.

CIGLIANO MM (1989) Revisión sistemática de la familia Tristiridae (Insecta: Orthoptera). Boletín de la Sociedad de Biología de Concepción (Chile) 60: 89-91.

CIGLIANO MM (1991) Congruencia taxonómica en la familia Tristiridae (Orthoptera, Acridoidea). Revista de la Sociedad Entomológica de Argentina 49: 85101 (1990)

CIGLIANO MM, RA RONDEROS \& WP KEMP (1989) Revisión del género Elasmoderus Saussure (Orthoptera: Tristiridae). Canadian Entomologist 121:225-243

CIGLIANO MM, WP KEMP \& T KALARIS (1995) Spatiotemporal characteristics of rangeland grasshopper (Orthoptera: Acrididae) regional outbreaks in Montana. Journal of Orthoptera Research 4: 111-126.

CLEMENTE M, GARCÍA MD \& JJ PRESA (1987) Clave de los géneros de saltamontes ibéricos (Orthoptera, Caelifera). Universidad de Murcia, Murcia, España. 64 pp.

CHAPPELL M (1983) Metabolism and thermoregulation in desert and montane grasshoppers. Oecologia 56: 126-131.

DALY HV (1985) Insect morphometrics. Annual Review of Entomology 30: 415-438.

DRAPER N \& H SMITH (1966) Applied regression analysis. John Wiley \& Sons, Inc., New York, USA. ix +407 pp.

ELGUETA M, A CAMOUSSEIGHT \& C CARBONELL (1999) Catálogo de los Orthoptera (Insecta) de Chile. Publicación Ocasional del Museo Nacional de Historia Natural (Chile) 54: 1-60.

FAIRBAIRN DJ (1990) Factors influencing sexual size dimorphism in temperate waterstriders. American Naturalist 136: 61-86.

FAIRBAIRN DJ (1993) Costs of loading associated with mate-carrying in the water strider, Aquarius remigis. Behavioral Ecology 4: 224-231.

FAIRBAIRN DJ (1997) Allometry for sexual dimorphism: pattern and process in the co-evolution of body sizes in males and females. Annual Review of Ecology and Systematics 28: 659-687.

FAIRBAIRN DJ \& RF PREZIOSI (1996) Sexual selection and the evolution of sexual size dimorphism in the water strider, Aquarius remigis. Evolution 50: 1549-1559.

GAGE SH \& MK MUKERJI (1977) A perspective of grasshopper population distribution in Saskatchewan and the interrelationship with weather. Environmental Entomology 6: 469-479.

GAJARDO R (1993) La vegetación natural de Chile: clasificación y distribución geográfica. Colección Imagen de Chile, Editorial Universitaria, Santiago, Chile. $165 \mathrm{pp}$.

GASTÓ J \& D CONTRERAS (1979) Un caso de desertificación en el norte de Chile. El ecosistema y su fitocenosis. Facultad de Agronomía, Universidad de Chile, Santiago, Chile, Boletín Técnico 42. 99 pp.

GREENWOOD PJ \& J ADAMS (1987) The ecology of sex. First edition. Edward Arnold Publishers Ltd., London, United Kingdom. 73 pp.

HEAD G (1995) Selection on fecundity and variation in the degree of sexual size dimorphism among spider species (Class Araneae). Evolution 49: 776-781.

HEDRICK AV \& EJ TEMELES (1989) The evolution of sexual dimorphism in animals: hypothesis and tests. Trends in Ecology and Evolution 4: 136-138.

HENSCHEL JR, MB ROBERTSON \& MK SEELY (2001) Animal ecophysiology in the Namib Desert: coping with little water, scarce food and elevated temperatures. En: Prakash I (ed) Ecology of desert environments: 423-457. Scientific Publishers, Jodhpur, India.

HIGGINS L (2000) Female gigantism in a New Guinea population of the spider Nephila maculate. Oikos 99: 377-385.

HILLIARD JR (2001) Two new grasshopper species in the texanus group of the genus Melanoplus (Orthoptera: Acrididae: Melanoplinae) with biological notes on the group. Transactions of the American Entomological Society 127: 31-68.

JABLONSKI P \& K VEPSÄLÄINEN (1995) Conflict between sexes in the water striders, Gerris lacustris: a test of two hypotheses for male guarding behavior. Behavioral Ecology 6: 388-392.

JAKOB EM, SD MARSHAL \& GW UETZ (1996) Estimating fitness: a comparison of body conditions indices. Oikos 77: 61-67.

JOERN A (1979) Resource utilization and community structure in assemblages of arid grassland grasshoppers (Orthoptera: Acrididae). Transactions American Entomology Society 105: 253-300.

KEMP WP (1992) Temporal variation in rangeland grasshoppers (Orthoptera: Acrididae) communities in the steppe region of Montana, USA. Canadian Entomologist 124: 437-450.

KOEHL M (1996) When does morphology matter? Annual Review of Ecology and Systematics 27: 501-542.

KRASNOV B, D WARD \& G SHENBROT (1996) Body length and leg variation in several species of darkling beetles (Coleoptera: Tenebrionidae) along a rainfall and altitudinal gradient in the Negev Desert (Israel). Journal of Arid Environments 34: 477-489.

LABARBERA M (1989) Analyzing body size as a factor in ecology and evolution. Annual Review of Ecology and Systematics 20: 97-117.

LACTIN DJ (1998) Environmental, physical and behavioral determinants of body temperature in grasshoppers nymphs (Orthoptera: Acrididae). Canadian Entomologist 130: 551-577. 
LANDE R (1980) Sexual dimorphism, sexual selection and adaptation in polygenic characters. Evolution 34: 292-207.

LIEBERMANN J (1954) Notas de ortopterología chilena, con la descripción de una nueva especie de Philippiacris Lieb., Ph. wagenknechti. Revista Universitaria (Chile) 39: 173-184.

LUZIO W \& S ALCAYAGA (1992) Mapa de asociaciones de grandes grupos de suelos de Chile. Agricultura Técnica (Chile) 52: 347-353.

MORONI J (1972) Irrupción de Elasmoderus rabiosus (Liebermann) en el Norte Chico (Acrididae, Chilacridinae). Noticiario Mensual del Museo Nacional de Historia Natural (Chile) 192: 3-6.

NOVOA R \& S VILLASECA (eds) (1989) Mapa agroclimático de Chile. Instituto de Investigaciones Agropecuarias, Santiago, Chile. $126 \mathrm{pp}$.

PALMER M (1994) Ecological factors associated with body size in populations of Macrothorax morbillosus (F) (Carabidae, Coleoptera). Acta Ecologica 15: 689-699.

PARKER GA (1978) Evolution of competitive mate searching. Annual Reviews of Entomology 23: 173-196.

PFADT RE (1977) Some aspects of the ecology of grasshopper populations inhabiting the short grass plains. En: Kulman HM \& HC Chiang (eds) Insect ecology: 73-79. University of Minnesota Agricultural Experimental Station, Technical Bulletin 310, Minnesota, USA

PFADT RE (1982) Density and diversity of grasshoppers (Orthoptera: Acrididae) in an outbreak on Arizona rangeland. Environmental Entomology 11: 690-694.

PFADT RE (1984) Species richness, density, and diversity of grasshoppers (Orthoptera: Acrididae) in a habitat of the mixed grass prairie. Canadian Entomologist 116:703-709.

PRANGE HG \& B PINSHOW (1994) Thermoregulation of an unusual grasshopper in a desert environment: the importance of food source and body size. Journal of Thermal Biology 19: 75-78.

PRENTER J, I MONTGOMERY \& RW ELWOOD (1995) Multivariate morphometrics and sexual dimorphism in the orb-web spider Metellina segmentata (Clerck, 1757) (Araneae, Metidae). Biological Journal of the Linnean Society 55: 345-354.

PREZIOSI RF \& DJ FAIRBAIRN (1996) Sexual selection and evolution of sexual dimorphism in the water strider, Aquarius remigis. Evolution 50: 1549-1559.

PREZIOSI RF \& DJ FAIRBAIRN (2000) Lifetime selection on adult body size and components of body size in a water strider: opposing selection and maintenance of sexual size dimorphism. Evolution 54: 558-566.

PREZIOSI RF \& DJ FAIRBAIRN, DA ROFF \& JM BRENNAN (1996) Body size and fecundity in the waterstriders, Aquarius remigis: a test of Darwin's fecundity advantage hypothesis. Oecologia 108: 424-431.

PRICE PW (1997) Insect ecology. Third edition. John Wiley and Sons Inc., New York, USA. 398 pp.

REEVE JP \& DJ FAIRBAIRN (1999) Change in sexual size dimorphism as a correlated response on fecundity. Heredity 83: 697-706.
REILLY SM \& PC WAINWRIGHT (1994) Conclusion: ecological morphology and the power of integration. En: Wainwright PC \& SM Reilly (eds) Ecological morphology. Integrative organismal biology: 339-354. University of Chicago Press, Chicago, Illinois, USA. 356 pp.

RICKLEFS RE \& DB MILES (1994) Ecological inferences from morphology; an ecological perspective. En: Wainwright PC \& SM Reilly (eds) Ecological morphology. Integrative organismal biology: 13-41. University of Chicago Press, Chicago, Illinois, USA.

ROURKE B (2000) Geographic and altitudinal variation in water balance and metabolic rate in a California grasshopper, Melanoplus sanguinipes. Journal of Experimental Biology 203: 2699-2712.

ROWE L (1994) The cost of mating and mate choice in water striders. Animal Behaviour 48: 1049-1056.

ROWE L \& G ARNQVIST (1996) Analysis of the causal components of assortative mating in water striders. Behavioral Ecology and Sociobiology 38: 279-286.

RUBENSTEIN DI (1989) Sperm competition in water strider, Gerris remigis. Animal Behaviour 38: 631-636.

SHINE R (1989) Ecological causes for the evolution of sexual dimorphism: a review of the evidence. Quaterly Review of Biology 64: 419-461.

SIVA-JOTHY MT (1987) Variation in copulation and the resultant degree of sperm removal in Orthetrum cancallatun (L) (Libellulidae: Odonata). Behavioral Ecology and Sociobiology 20: 147-151.

SLATKIN M (1984) Ecological causes of sexual dimorphism. Evolution 38: 622-630.

SQUEO F, G ARANCIO, C MARTICORENA, M MUÑOZ \& JG GUTIÉRREZ (2002) Diversidad vegetal en la IV Región de Coquimbo, Chile. En: Squeo FA, G Arancio \& JR Gutiérrez (eds) Libro rojo de la flora nativa y de los sitios prioritarios para su conservación: Región de Coquimbo: 149158. Ediciones Universidad de La Serena. La Serena, Chile.

THOMPSON DB (1992) Consumption rates and the evolution of diet-induced plasticity in the head morphology of Melanoplus femurrubrum (Orthoptera: Acrididae.). Oecologia 89: 204-213.

TORO H (1972) Notas biológicas sobre la langosta de Combarbalá, Philippiacris rabiosus (Phil). Idesia (Chile) 2: 133-136.

VILLAGRA CA, CA VILLALOBOS, DH TAPIA \& K RODRIGUEZ-AUAD (2001) Sexual dimorphism and behavior in the water strider, Gerris chilensis (Berg) (Hemiptera: Gerridae). Revista Chilena de Entomología 28: 87-93.

WAINWRIGHT PC (1994) Functional morphology as a tool in ecological research. En: Wainwright PC \& SM Reilly (eds) Ecological morphology. Integrative organismal biology: 42-59. University of Chicago Press, Chicago, Illinois, USA.

WILLIAMS BL (2001) Patterns 1356 pp of morphological variation in Speyeria idalia (Lepidoptera: Nymphalidae) with implications for taxonomy and conservation. Annals of the Entomological Society of America 94: 239-243. 